\title{
HOMOTOPY GROUPS OF COMPACT LIE GROUPS $\mathbf{E}_{6}, \mathrm{E}_{7}$ AND $\mathbf{E}_{8}$
}

\section{HIDEYUKI KACHI}

\section{$\S 1$. Introduction}

Let $G$ be a simple, connected, compact and simply-connected Lie group. If $k$ is the field with characteristic zero, then the algebra of cohomology $H^{*}(G ; k)$ is the exterior algebra generated by the elements $x_{1}, \cdots, x_{l}$ of the odd dimension $n_{1}, \cdots, n_{l}$; the integer $l$ is the rank of $G$ and $n=\sum_{i=1}^{l} n_{i}$ is the dimension of $G$. Let $X$ be the direct product of spheres of dimension $n_{1}, \cdots, n_{l}$, then there exists a continuous map $f: G \longrightarrow X$ which induces isomorphisms of $H^{i}(X ; k)$ to $H^{i}(G ; k)$ for all $i$ (cf. [8]). From this we deduce by Serre's $C$-theory [8] that $f_{*}: \pi_{i}(G) \longrightarrow \pi_{i}(X)$ are $C$-isomorphisms. for all $i$, where $C$ is the class of finite abelian groups. Therefore the rank of $\pi_{q}(G)$ is equal to the number of such $i$ that $n_{i}$ is equal to $q$, and particularly if $q$ is even, then $\pi_{q}(G)$ is finite. It is a classical fact that $\pi_{2}(G)=0$ and $\pi_{3}(G)=Z$.

According to Bott-Samelson [6] ;

$$
\begin{array}{lll}
\pi_{i}\left(E_{6}\right)=0 & \text { for } 4 \leqslant i \leqslant 8, & \pi_{9}\left(E_{6}\right)=Z, \\
\pi_{i}\left(E_{7}\right)=0 & \text { for } 4 \leqslant i \leqslant 10, & \pi_{11}\left(E_{7}\right)=Z, \\
\pi_{i}\left(E_{8}\right)=0 & \text { for } 4 \leqslant i \leqslant 14, & \pi_{15}\left(E_{8}\right)=Z .
\end{array}
$$

where $E_{6}, E_{7}$ and $E_{8}$ are compact exceptional Lie groups.

In this paper, using the killing method we compute the 2-components of homotopy group $\pi_{j}(G)$, where $G=E_{6}, E_{7}$ and $E_{8}$. The resuls are stated as follows;

\begin{tabular}{|c|c|c|c|c|c|c|c|c|c|c|}
\hline$j$ & $4 \leqslant j \leqslant 14$ & 15 & 16 & 17 & 18 & 19 & 20 & 21 & 22 & 23 \\
\hline$\pi_{j}\left(E_{8}: 2\right)$ & 0 & $Z$ & $Z_{2}$ & $Z_{2}$ & $Z_{8}$ & 0 & 0 & $Z_{2}$ & 0 & $Z+Z_{2}$ \\
\hline
\end{tabular}

\begin{tabular}{|c|c|c|c|c|c|}
\hline$j$ & 24 & 25 & 26 & 27 & 28 \\
\hline$\pi_{j}\left(E_{8}: 2\right)$ & $Z_{2}+Z_{2}$ & $Z_{2}$ & 0 & $Z$ & 0 \\
\hline
\end{tabular}

Received April 3, 1967. 


\begin{tabular}{|c|c|c|c|c|c|c|c|c|c|c|}
\hline$j$ & $4 \leqslant j \leqslant 10$ & 11 & 12 & 13 & 14 & 15 & 16 & 17 & 18 & 19 \\
\hline$\pi_{j}\left(E_{7}: 2\right)$ & 0 & $Z$ & $Z_{2}$ & $Z_{2}$ & 0 & $Z$ & $Z_{2}$ & $Z_{2}$ & $Z_{4}$ & $Z+Z_{2}$ \\
\hline
\end{tabular}

\begin{tabular}{|c|c|c|c|c|c|c|}
\hline$j$ & 20 & 21 & 22 & 23 & 24 & 25 \\
\hline$\pi_{j}\left(E_{7}: 2\right)$ & $Z_{2}$ & $Z_{2}$ & $Z_{4}$ & $Z_{2}+Z_{2}+Z_{2}$ & $Z_{2}+Z_{2}+Z_{2}$ & $Z_{2}+Z_{2}$ \\
\hline
\end{tabular}

\begin{tabular}{|c|c|c|c|c|c|c|c|c|c|c|}
\hline$j$ & $4 \leqslant j \leqslant 8$ & 9 & 10 & 11 & 12 & 13 & 14 & 15 & 16 & 17 \\
\hline$\pi_{j}\left(E_{6}: 2\right)$ & 0 & $Z$ & 0 & $Z$ & $Z_{4}$ & 0 & 0 & $Z$ & 0 & $Z+Z_{2}$ \\
\hline
\end{tabular}

\begin{tabular}{|c|c|c|c|c|c|}
\hline$j$ & 18 & 19 & 20 & 21 & 22 \\
\hline$\pi_{j}\left(E_{6}: 2\right)$ & $Z_{16}+Z_{2}$ & 0 & $Z_{8}$ & 0 & 0 \\
\hline
\end{tabular}

All spaces that we concider in this paper are those which have the homotopy groups of finite type. Let $G$ be such a space, then $\pi_{i}(G)$ is isomorphic to the direct sum of a free part $F$ and the $p$-components of $\pi_{i}(G)$ for every prime $p$. We denote by $\pi_{i}(G: p)$ the direct sum of a certain subgroup $F^{\prime}$ of $F$ and the $p$-component of $\pi_{i}(G)$, where the index $\left[F ; F^{\prime}\right]$ is prime to $p$.

Given an exact sequence for such $A, B$ and $C$

$$
\cdots \longrightarrow \pi_{i}(A) \longrightarrow \pi_{i}(B) \longrightarrow \pi_{i}(C) \longrightarrow \cdots,
$$

then we can form the following exact one in our case

$$
\cdots \longrightarrow \pi_{i}(A: p) \longrightarrow \pi_{i}(B: p) \longrightarrow \pi_{i}(C: p) \longrightarrow \cdots
$$

The author is indebted to Professor H. Toda for his advice during the preparation of the paper.

\section{$\S 2$. The cohomology of the 3 -connective fibre spaces of $E_{6}, E_{7}$ and $\boldsymbol{E}_{8}$.}

H. Cartan and J.P. Serre introduced a method to calculate the homotopy group in [7]. Let $K(\pi, n)$ be an Eilenberg-Mac-Lane space of type $(\pi, n)$.

Theorem 2.1. Let $X$ be an arcwise connected topological space, then there exists a sequence of $(n-1)$-connected spaces $(X, n)(n=1,2, \cdots, \quad$ and $(X, 1)=X)$ and continuous maps $f_{n}:(X, n+1) \longrightarrow(X, n)$ such that: 
(I) the triple $\left((X, n+1), f_{n},(X, n)\right)$ is a fibre space with a fibre $K\left(\pi_{n}(X), n-1\right)$. (II) there exists a fibre space $X_{n}^{\prime}$ over the base space $K\left(\pi_{n}(X), n\right)$, where $X_{n}^{\prime}$ and $(X, n)$ are of the same homotopy type, such that the fibre is $(X, n+1)$.

Hence $f_{1} \circ f_{2} \circ \cdots \circ f_{n-1}$ defines the isomorphism of $\pi_{i}(X, n)$ to $\pi_{i}(X)$ for $i \geqslant n$.

Lemma 2. 2. Let $X$ be a 2-connected topological space. Assume that $X$ satisfies the following conditions,

(1) $\pi_{3}(X)$ is isomorphic to an infinite cyclic group,

(2) $H^{*}\left(X ; Z_{2}\right)=A_{0} \otimes A_{1} \otimes \cdots \otimes A_{r} \otimes B$

where $x_{3}$ is a generator of $H^{3}\left(X ; Z_{2}\right) \approx Z_{2}, A_{0}=Z_{2}\left[x_{3}\right] /\left(x_{3}\right)^{s_{0}}, A_{i}=Z_{2}\left[S q^{2^{i}} S q^{2^{i-1}} \ldots\right.$ $\left.S q^{2} x_{3}\right] /\left(S q^{2 i} S q^{2^{i-1}} \cdots S q^{2} x_{3}\right)^{2^{s_{i}}}\left(s_{i} \geqslant 1\right) \quad 1 \leqslant i \leqslant r$, and $S q^{2^{r+1}} S q^{2 r} \cdots S q^{2} x_{3}=0$, then

$$
H^{*}\left((X, 4) ; Z_{2}\right)=Z_{2}[w] \otimes \Delta\left(a_{0}, a_{1}, \cdots, a_{r}\right) \otimes B^{\prime}
$$

where the deg. $a_{i}=\left(2^{i+1}+1\right)\left(2^{s_{i}}-1\right)+2^{2^{i}}$, deg.w $=2^{2^{r+1}}, \Delta\left(a_{0}, a_{1}, \cdots, a_{r}\right)$ indicates a submodule having $a_{0}, \cdots, a_{r}$ as a simple system of generators and $B^{\prime}$ is isomorphic to $B$ by $\left(f_{1} \circ f_{2} \circ f_{3}\right)^{*}: H^{*}\left(X ; Z_{2}\right) \longrightarrow H^{*}\left((X, 4) ; Z_{2}\right)$.

Proof. From the above theorem, there exists a fibre space $((X, 4)$, $\left.f_{1} \circ f_{2} \circ f_{3}, X\right)$ with a fibre $K(Z, 2)$. Since $K(Z, 2)$ is the infinite dimensional complex projective space, its $\bmod 2$ cohomology structure is $H^{*}\left(Z, 2 ; Z_{2}\right)$ $\approx Z_{2}[u]$, where $u$ is a generator of $H^{2}\left(Z, 2 ; Z_{2}\right)$. Let $\left\{E_{r}^{* *}\right\}$ be the $\bmod 2$ spectral sequence associated to the above fibration $((X, 4), X, K(Z, 2))$, then

$$
E_{2}^{* *}=A_{0} \otimes A_{1} \otimes \cdots \otimes A_{r} \otimes B \otimes Z_{2}[u] .
$$

Clearly we have $d_{3}(1 \otimes u)=x_{3} \otimes 1$. Hence if $n$ is even, $d_{3}\left(1 \otimes u^{n}\right)=0$, if $n$ is odd, $\quad d_{3}\left(1 \otimes u^{n}\right)=x_{3} \otimes u^{n-1}, \quad$ and $\quad d_{3}\left(x_{3}^{s_{0}-1} \otimes u^{n}\right)=0$ for all $n>0$. Thus we obtain

$$
E_{4}^{* *}=\Lambda\left(\bar{a}_{0}\right) \otimes A_{1} \otimes A_{2} \otimes \cdots \otimes A_{r} \otimes B \otimes Z_{2}\left[u^{n}\right]
$$

where $\bar{a}_{0}=\left(x_{3}\right)^{s_{0}-1} \otimes u$.

Let $\tau$ be the transgression, $\tau\left(u^{2}\right)=S q^{2} x_{3}$, since the transgression commutes the Steenrod operation. Thus $d_{5}\left(1 \otimes u^{2}\right)=S q^{2} x_{3} \otimes 1$. Since $d_{t}$ is derivative, $\quad d_{5}\left(1 \otimes u^{2 n}\right)=0$ if $n$ is even, $d_{5}\left(1 \otimes u^{2 n}\right)=S q^{2} x_{3} \otimes u^{2(n-1)}$ if $n$ is odd, and $d_{5}\left(\left(S q^{2} x_{3}\right)^{2^{s_{i}-1}} \otimes u^{2 n}\right)=0$ for all $n \geqslant 1$. Thus 


$$
E_{6}^{* *}=\Lambda\left(\bar{a}_{0}, \bar{a}_{1}\right) \otimes A_{2} \otimes A_{3} \otimes \cdots \otimes A_{r} \otimes B \otimes Z_{2}\left[u^{4}\right]
$$

where $\vec{a}_{1}=\left(S q^{2} x_{3}\right)^{2 s_{i}-1} \otimes u^{2}$.

Carrying on similarly, we have

$$
E_{2^{++1}+2}^{* *}=\Lambda\left(\bar{a}_{0}, \bar{a}_{1}, \cdots, \bar{a}_{r}\right) \otimes B \otimes Z_{2}\left[u^{2^{r+1}}\right]
$$

where $\bar{a}_{i}=\left(S q^{2 i} S q^{2^{i-1}} \cdots S q^{2} x_{3}\right)^{2^{s_{i}-1}} \otimes u^{2^{i}}, i=0,1, \cdots, r$, and $s_{i} \geqslant 1$. Clearly $d_{t}=0$ for all $t \geqslant 2^{r+1}+2$. Thus we obtain

$$
E_{\infty}^{* *}=\Lambda\left(a_{0}, a_{1}, \cdots, a_{r}\right) \otimes B \otimes Z_{2}\left[u^{2^{r+1}}\right] .
$$

Since $E_{\infty}^{* *}$ is the graded algebra associated to $H^{*}\left((X, 4) ; Z_{2}\right)$, assume that $a_{i}, w, B^{\prime}$ correspond to $\bar{a}_{i}, u^{2 r+1}, B$ respectively. We have the lemma.

Particularly, we can assume that $B$ is mapped isomorphically onto $B^{\prime}$ by the homomorphism $\left(f_{1} \circ f_{2} \circ f_{3}\right)^{*} ; H^{*}\left(X ; Z_{2}\right) \longrightarrow H^{*}\left((X, 4) ; Z_{2}\right)$. Thus the relation of $B$ are arranged in $B^{\prime}$.

The mod 2 cohomology algebra of the exceptional Lie groups have been determined by S. Araki [2] and S. Araki-Y. Shikata [3]. These algebra are as follow.

$$
\begin{aligned}
& H^{*}\left(F_{4} ; Z_{2}\right)=Z_{2}\left[x_{3}\right] /\left(x_{3}^{4}\right) \otimes \Lambda\left(S q^{2} x_{3}, x_{15}, S q^{8} x_{15}\right), \\
& H^{*}\left(E_{6} ; Z_{2}\right)=Z_{2}\left[x_{3}\right] /\left(x_{3}^{4}\right) \otimes \Lambda\left(S q^{2} x_{3}, S q^{4} S q^{2} x_{3}, x_{15}, S q^{8} S q^{4} S q^{2} x_{3}, S q^{8} x_{15}\right) \text {, } \\
& H^{*}\left(E_{7} ; Z_{2}\right)=Z_{2}\left[x_{3}, S q^{2} x_{3}, S q^{4} S q^{2} x_{3}\right] /\left(x_{3}^{4},\left(S q^{2} x_{3}\right)^{4},\left(S q^{4} S q^{2} x_{3}\right)^{4}\right) \\
& \otimes \Lambda\left(x_{15}, S q^{8} S q^{4} S q^{2} x_{3}, S q^{8} x_{15}, S q^{4} S q^{8} x_{15}\right) \text {, } \\
& H^{*}\left(E_{8} ; Z_{2}\right)=Z_{2}\left[x_{3}, S q^{2} x_{3}, S q^{4} S q^{2} x_{3}, x_{15}\right] /\left(x_{3}^{16},\left(S q^{2} x_{3}\right)^{8},\left(S q^{4} S q^{2} x_{3}\right)^{4}, x_{15}^{4}\right) \\
& \otimes \Lambda\left(S q^{8} S q^{4} S q^{2} x_{3}, S q^{8} x_{15}, S q^{4} S q^{8} x_{15}, S q^{2} S q^{4} S q^{8} x_{15}\right)
\end{aligned}
$$

where $x_{i}$ denotes a generator of degree $i$.

(2. 5) In the inclusion $F_{4} \subset E_{6} \subset E_{7} \subset E_{8}$, every subgroup is totally nonhomologous to zero mod 2 in any bigger group containing it, where each exceptional group denotes simply-connected one. (See, S. Araki and Y. Shikata [3], Theorem 3).

If $S q^{16} S q^{8} S q^{4} S q^{2} x_{3}=0$ in $E_{8}$, then this is a primitive element. By (2.4), there is no primitive element of degree 33. Thus $S q^{16} S q^{8} S q^{4} S q^{2} x_{3}=0$ in $E_{8}$. Similarly we have $S q^{16} S q^{8} S q^{4} S q^{2} x_{3}=0$ in $E_{6}, E_{7}$ and $S q^{4} S q^{2} x_{3}=0$ in $F_{4}$.

Corollary 2.3. Let $\tilde{G}$ be the 3-connective fibre space over $G$ : where $G=F_{4}, E_{6}, E_{7}, E_{8}$, then 


$$
\begin{aligned}
& H^{*}\left(\widetilde{F}_{1} ; Z_{2}\right)=Z_{2}\left[y_{8}\right] \otimes \Delta\left(y_{9}, y_{11}, y_{15}, y_{23}\right), \\
& H^{*}\left(\widetilde{E}_{6} ; Z_{2}\right)=Z_{2}\left[y_{32}\right] \otimes \Delta\left(y_{9}, y_{11}, y_{15}, y_{17}, y_{23}, y_{33}\right), \\
& H^{*}\left(\widetilde{E}_{7} ; Z_{2}\right)=Z_{2}\left[y_{32}\right] \otimes \Delta\left(y_{11}, y_{15}, y_{19}, y_{23}, y_{27}, y_{33}, y_{35}\right), \\
& H^{*}\left(\widetilde{E}_{8} ; Z_{2}\right)=Z_{2}\left[y_{15}, y_{32}\right] /\left(y_{15}^{4}\right) \otimes \Delta\left(y_{23}, y_{27}, y_{29}, y_{33}, y_{35}, y_{39}, y_{47}\right),
\end{aligned}
$$

where $y_{i}$ denotes a generator of degree $i$. By the naturality of the homomorphism $p^{*}=\left(f_{1} f_{2} f_{3}\right)^{*}$, we have

$$
\begin{array}{ll}
S q^{8} y_{15}=y_{23} & \text { in } \tilde{E}_{6}, \tilde{E}_{7}, \tilde{E}_{8} \text { and } \tilde{F}_{4}, \\
S q^{4} y_{23}=y_{27} & \text { in } \tilde{E}_{7}, \tilde{E}_{8}, \\
S q^{2} y_{27}=y_{29} & \text { in } \tilde{E}_{8} .
\end{array}
$$

Lемма 2. 4. We have the following relations,

$$
\begin{aligned}
& S q^{1} y_{8}=y_{9}, S q^{2} y_{9}=y_{11} \quad \text { in } \tilde{F}_{4}, \\
& S q^{2} y_{9}=y_{11}, S q^{8} y_{9}=y_{17} \quad \text { in } \tilde{E}_{6}, \\
& S q^{8} y_{11}=y_{19} \quad \text { in } \tilde{E}_{7} .
\end{aligned}
$$

Proof. (i) From Theorem 2. 1, there exists a fibration $\left(\bar{F}_{4}, K(Z, 3), \widetilde{F}_{4}\right)$, where $\bar{F}_{4}$ denotes the space which has same homotopy type as $F_{4}$. We consider the spectral sequence $\left\{E_{r}^{* *}\right\}$ over $Z_{2}$ associated with the above fibration. Then

$$
E_{2}^{* *}=H^{*}\left(Z, 3 ; Z_{2}\right) \otimes H^{*}\left(\tilde{F}_{4} ; Z_{2}\right)
$$

It is known that

$$
H^{*}\left(Z, 3 ; Z_{2}\right)=Z_{2}\left[v, S q^{2} v, S q^{4} S q^{2} v, \cdots\right]
$$

where $v$ is a fundamental class of $H^{3}\left(Z, 3: Z_{2}\right)$. From the $\bmod 2$ cohomology algebra of $F_{4}, S q^{4} v \otimes 1,\left(S q^{2} v\right)^{2} \otimes 1$ and $v^{4} \otimes 1$ must be $d_{r}$-images for some $r$. If $p \neq 0$ and $0<q<8$, or $q \neq 0$ and $0<p<3$, then $E_{r}^{p, q}=0$ for all $r$. Since $E_{r}^{0.8}$ has only one element $1 \otimes y_{8}$ for $r \leqslant 9, \quad S q^{4} S q^{2} v \otimes 1$ is not a $d_{r}$-image for $r \leqslant 8$. Thus $\tau$ be the transgression, we have $\tau\left(y_{8}\right)=S q^{4} S q^{2} v$. Since $E_{r}^{0, \theta}$ has only one generator $1 \otimes y_{9}$ and $\left(S q^{2} v\right)^{2} \otimes 1$ is not a $d_{r}$-image for $r \leqslant 10$, we have that $\tau\left(y_{9}\right)=\left(S q^{2} v\right)^{2}$. Consider

$$
d_{r}: E_{r}^{p, q} \longrightarrow E_{r}^{12.0} \quad \text { for } p+q=11 \text { and } r=q+1 \text {. }
$$

From Corollary 2. 3, we have $E_{r}^{p, q}=0$ for $q \neq 8,9$. But $E_{r}^{2,9}=0$. $E_{r}^{3,8}$ has one generator $\quad v \otimes y_{8}$ and $d_{9}\left(v \otimes y_{8}\right)=v S q^{4} S q^{2} v \otimes 1 \neq 0$, for $d_{9}\left(1 \otimes y_{8}\right)$ 
$=S q^{4} S q^{2} v \otimes 1$. Thus $E_{12}^{0,11}$ has only one generator $1 \otimes y_{11}$ and $v^{4} \otimes 1$ is not a $d_{r}$-image for $r \leqslant 11$. Therefore we have that $\tau\left(y_{11}\right)=v^{4}$. Using Adem's relation, from $S q^{1} S q^{4} S q^{2} v=S q^{5} S q^{2} v=\left(S q^{2} v\right)^{2}, S q^{2}\left(S q^{2} v\right)^{2}=S q^{2} S q^{5} S q^{2} v$ $=S q^{6} S q^{3} v=v^{4}$, we obtain $S q^{1} y_{8}=y_{9}$, and $S q^{2} y_{9}=y_{11}$.

(ii) From Theorem 2. 1, there exists a fibration $\left(\bar{E}_{6}, K(Z, 3), \widetilde{E}_{6}\right)$ where $\bar{E}_{6}$ denotes the space which has the same homotopy type as $E_{6}$. Let $\tau$ be the transgression associated with this fibration. Let $\left\{E_{r}^{p, q}\right\}$ be the $\bmod 2$ spectral sequence associated with this fibration. Then

$$
E_{2}^{* *}=H^{*}\left(Z, 3 ; Z_{2}\right) \otimes H^{*}\left(\widetilde{E}_{6} ; Z_{2}\right) .
$$

By the same argument as in $\widetilde{F}_{4}$, we have that $\tau\left(y_{9}\right)=\left(S q^{2} v\right)^{2}$ and $\tau\left(y_{11}\right)=v^{4}$. Concider

$$
d_{r} ; E_{r}^{p, q} \longrightarrow E_{r}^{18,0} \text { for } p+q=17 \text { and } r=q+1 .
$$

From Corollary 2. 3, we have $E_{r}^{p, q}=0$ for $q \neq 9,11,15$ and $17 \quad(q \leqslant 22)$. But $E_{r}^{2,1 \sigma}=0 . \quad E_{10}^{8,9}$ has one generator $\left(v S q^{2} v\right) \otimes y_{9}$ and $d_{10}\left(\left(v S q^{2} v\right) \otimes y_{9}\right)$ $=v\left(S q^{2} v\right)^{3} \otimes 1 \neq 0, \quad$ for $\quad d_{10}\left(1 \otimes y_{9}\right)=\left(S q^{2} v\right)^{2} \otimes 1 . \quad E_{12}^{8.11}$ has one generator $v^{2} \otimes y_{11}$ and $d_{12}\left(v^{2} \otimes y_{11}\right)=v^{6} \otimes 1 \neq 0$ for $d_{12}\left(1 \otimes y_{11}\right)=v^{4} \otimes 1$. Thus, since $E_{17}^{0,17}$ has one generator $y_{17}$ and $\left(S q^{4} S q^{2} v\right)^{2} \otimes 1$ is not a $d_{r}$-image for $r \leqslant 16, \quad d_{18}\left(1 \otimes y_{17}\right)=\left(S q^{4} S q^{2} v\right)^{2} \otimes 1, \quad$ i.e. $\quad \tau\left(y_{17}\right)=\left(S q^{4} S q^{2} v\right)^{2}$. Using Adem's relation, $S q^{2}\left(S q^{2} v\right)^{2}=S q^{2} S q^{5} S q^{2} v=S q^{6} S q^{3} v=v^{4} \quad$ and $S q^{8}\left(S q^{2} v\right)^{2}=S q^{8} S q^{5} S q^{2} v$ $=S q^{9} S q^{4} S q^{2} v=\left(S q^{4} S q^{2} v\right)^{2}$. From the commutativity of the Steenrod operation and the transgression, we obtain $S q^{2} y_{9}=y_{11}$ and $S q^{8} y_{9}=y_{17}$.

(iii) Consider the fibration $\left(\bar{E}_{7}, K(Z, 3), \widetilde{E}_{7}\right)$ of theorem 2.1 (II), where $\bar{E}_{7}$ has the same homotopy type as $E_{7}$. Let $\left\{E_{r}^{p, q}\right\}$ be the mod 2 spectral sequence associated with this fibration. Then

$$
E_{r}^{* *}=H^{*}\left(Z, 3 ; Z_{2}\right) \otimes H^{*}\left(\widetilde{E}_{7} ; Z_{2}\right)
$$

From the mod 2 cohomology algebra of $E_{7}, v^{4} \otimes 1$ and $\left(S q^{2} v\right)^{4} \otimes 1$ must be the $d_{r}$-images for some $r$. Since $H^{*}\left(\bar{E}_{7} ; Z_{2}\right)=0$ for degree $\leqslant 10$, we have $E_{r}^{p, q}=0$ for $p \neq 0$ and $0<q<10$. Thus we have that $\tau\left(y_{11}\right)=v^{4}$, where $\tau$ is the transgression. Consider

$$
d_{r} ; E_{r}^{p, q} \longrightarrow E_{r}^{20,0} \quad \text { for } p+q=19 \text { and } r=q+1 .
$$

From $H^{2}\left(\widetilde{E}_{7} ; Z_{2}\right)=0$ for $i \neq 11,15$ and $i<19$, it follow that $E_{r}^{p, q}=0$ for $(p, q) \neq(4,11)$ and $(2,15)$. On the other hand $H^{i}\left(Z, 3 ; Z_{2}\right)=0$ for $i=2,4$ 
and $i \leqslant 4$. Thus $E_{r}^{p, q}=0$ for $(p, q)=(4,11)$ and $(2,15)$. From this we obtain $\tau\left(y_{19}\right)=\left(S q^{2} v\right)^{4}$. By Adem's relation $S q^{8} v^{4}=S q^{8} S q^{6} S q^{3} v=S q^{10} S q^{4} S q^{3} v$ $+S q^{11} S q^{3} S q^{3} v=S q^{10} S q^{5} S q^{2}+S q^{11} S q^{5} S q^{1} v=\left(S q^{2} v\right)^{4}$. Thus we obtain $S q^{8} y_{11}$ $=y_{19}$.

LEMma 2.5. Let a topological space $X$ be 2-connected and the homology of finite type. Assume that $H^{*}\left(X ; Z_{2}\right)$ has the additive basis $a_{1}, \cdots, a_{s}$ for dim. $<N$. Then there exist a finite cell complex $K={ }_{*} \cup e_{1} \cup e_{2} \cup \cdots \cup e_{s}$, where dim. $e_{i}$ $=$ degree $a_{i}=n_{i}$ and a continuous map $f ; K \longrightarrow X$ such that $f$ induces isomorphism of $H^{*}\left(X ; Z_{2}\right)$ onto $H^{*}\left(K ; Z_{2}\right)$ for dim. $<N$.

Particularly if $\pi_{n_{i}-1}\left(K^{n_{i}-1}\right)$ is finite, then we can assume that the class of attaching map of $e_{i}$ belong to the 2-components. Here $*$ denotes a vertex and $K^{n}$ the $n$-skelton of $K$.

Proof. We prove this by induction on dimension $N$. Suppose that there exist a finite cell complex $K_{0}=K^{N-1}$ and a continuous map $f_{0} ; K_{0}$ $\longrightarrow X$ satisfying lemma 2.5 for $\operatorname{dim} .<N$. Here we may assume that $f_{0} ; K_{0} \longrightarrow X$ is the injection by the mapping-cylinder argument. Suppose that $H^{N}\left(X ; Z_{2}\right)$ has generator $a_{s+1}, \cdots, a_{r}$.

From the cohomology exact sequence for pair $\left(X, K_{0}\right)$ and the assumption of the induction, we have

$$
\begin{aligned}
& H^{i}\left(X, K_{0} ; Z_{2}\right)=0 \quad \text { for } i<N, \\
& H^{N}\left(X, K_{0} ; Z_{2}\right) \approx H^{N}\left(X ; Z_{2}\right) .
\end{aligned}
$$

By the duality, we obtain

$$
H_{i}\left(X, K_{0} ; Z_{2}\right)=0 \quad \text { for } i<N
$$

and

$$
H_{N}\left(X, K_{0} ; Z_{2}\right) \text { has the generators } \bar{a}_{s+1}, \cdots, \bar{a}_{r} \text {. }
$$

By Serre's C-theory [8], we have that $\pi_{N}\left(X, K_{0}\right) \otimes Z_{2} \longrightarrow H_{N}\left(X, K_{0}\right)$ $\otimes Z_{2}$ is an isomorphism. Let $f_{i}:\left(E^{N}, S^{N-1}\right) \longrightarrow\left(X, K_{0}\right)(i=1,2, \cdots, r-s)$ be the generators of $\pi_{N}\left(X, K_{0}\right)$ such that they correspond to $\bar{a}_{s+i}$ by the above isomorphism and construct a cell complex $K$ which is obtained from the disjoint union of $C\left(S_{1}^{N-1} \bigvee \cdots \vee S_{r-s}^{N-1}\right)$ and $K_{0}$ by identifying $S_{1}^{N-1} \bigvee \cdots \vee S_{r-s}^{N-1}$ with its image under a map $\left(f_{1} \mid S_{1}^{N-1}\right) \bigvee \cdots \vee\left(f_{r-s} \mid S_{r-s}^{N-1}\right)$; $S_{1}^{N-1} \vee \cdots \vee S_{r-8}^{N-1} \longrightarrow K_{0}$, where $C Y$ is a cone over the space $Y$ and $S_{i}^{N-1}$ is a $(N-1)$-sphere. Using the map $f_{i}$ the inclusion map $f_{0} ; K_{0} \longrightarrow X$ has 
an extension over $K$ and we denote this extension by $g: K \longrightarrow X$. Then $g: K \longrightarrow X$ induce an isomorphism $H_{N}\left(K, K_{0} ; Z_{2}\right)$ onto $H_{N}\left(X, K_{0} ; Z_{2}\right)$ and from the duality between homology and cohomology, it follows that $g^{*}$ : $H^{N}\left(X, K_{0} ; Z_{2}\right) \longrightarrow H^{N}\left(K, K_{0} ; Z_{2}\right)$ is an isomorphism onto.

Applying the five lemma to the diagram

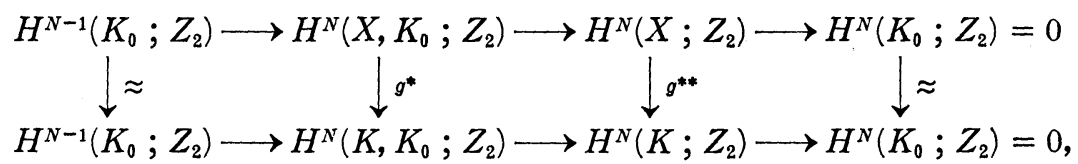

we obtain that

$$
g^{*}: H^{N}\left(X ; Z_{2}\right) \longrightarrow H^{N}\left(K ; Z_{2}\right)
$$

is an isomorphism.

Particularly if $\pi_{N-1}\left(K_{0}\right)$ is finite, then there exists an odd integer $q$ such that $q\left\{f_{i} \mid S^{N-1}\right\}$ belongs to the 2-component of $\pi_{N-1}\left(K_{0}\right)$. Displacing $f_{i}$ by $q f_{i}$, it is sufficient for the last statement that we construct a cell complex $K$ from $K_{0}$. Consequently the lemma is proved.

Let $\alpha$ be an element of $\pi_{n+i-1}\left(S^{n}\right)$ and consider a cell complex $K_{\alpha}=S^{n} \bigcup_{\alpha} e^{n+i}$ which is uniquely determined by $\alpha$ up to homotopy type.

Theorem 2. 6. Let $n>i$ and $i=2$ (4 or 8 respectively), then $S q^{i}$ : $H^{n}\left(K_{\alpha} ; Z_{2}\right) \longrightarrow H^{n+i}\left(K_{\alpha} ; Z_{2}\right)$ is an isomorphism onto if and only if $\alpha \equiv \eta_{n}$, ( $\nu_{n}$ or $\sigma_{n}$ respectively) mod $2 \pi_{n+i-1}\left(S^{n}\right)$. (For the proof see H. Tada; [11] Proposition 8. 1)

From Lemma 2.5 and Corollary 2.3, there exist a cell complex $M=S^{8} \cup e^{9} \cup e^{11} \cup e^{15}$ and a continuous map $f: M \longrightarrow \tilde{F}_{4}$ such that $f$ induces an $C_{2}$-isomorphisms $\pi_{i}(M)$ onto $\pi_{i}\left(\tilde{F}_{4}\right)$ for $i \leqslant 14$, where $C_{2}$ is the classes of finite abelian group whose 2-primary components are zero. Since $S q^{1} y_{8}=y_{9}$ in $\tilde{F}_{4}$, we may assume that $e^{9}$ is attached to $S^{8}$ by a map of degree two. Then we have

$$
\begin{aligned}
& \pi_{13}\left(S^{8} \underset{2}{\cup} e^{9}: 2\right)=0, \\
& \pi_{14}\left(S^{8} \bigcup_{2} e^{9}: 2\right) \approx \pi_{14}\left(S^{8}: 2\right)=Z_{2} \quad \text { generated by } \nu_{8}^{2},
\end{aligned}
$$

we denote by $\nu_{8}^{2}$ a generator of $\pi_{14}\left(S^{8} \bigcup_{2} e^{9}: 2\right)$ identifying with that of $\pi_{14}\left(S^{8}: 2\right)$ by the inclusion $S^{8} \subset S^{8} \bigcup_{2} e^{9}$.

Consider the following exact sequence 


$$
\pi_{i}\left(S^{8}: 2\right) \longrightarrow \pi_{i}\left(S^{8}: 2\right) \longrightarrow \pi_{i}\left(S^{8} \cup e^{9}: 2\right) \longrightarrow \pi_{i}\left(S^{9}: 2\right) \longrightarrow \pi_{i}\left(S^{9}: 2\right)
$$

for $i \leqslant 15$. From $\pi_{12}\left(S^{8}\right)=\pi_{13}\left(S^{9}\right)=\pi_{14}\left(S^{9}\right)=0$ and $\pi_{14}\left(S^{8}\right)=\left\{\nu_{8}^{2}\right\}=Z_{2}$, (2. 6$)$ is obtained.

Consider the exact sequence

$$
\begin{aligned}
\pi_{14}\left(S^{10}: 2\right) & \longrightarrow \pi_{14}\left(S^{8} \bigcup_{2} e^{9}: 2\right) \stackrel{i^{*}}{\longrightarrow} \pi_{14}\left(S^{8} \bigcup_{2} e^{9} \cup e^{11}: 2\right) \stackrel{j_{*}}{\longrightarrow} \pi_{14}\left(S^{11}: 2\right) \\
& \longrightarrow \pi_{14}\left(S^{9} \underset{2}{\cup} e^{10}: 2\right)
\end{aligned}
$$

where $i$ is the inclusion $S^{8} \bigcup_{2} e^{9} \subset S^{8} \bigcup_{2} e^{9} \cup e^{11}$, and $j: S^{8} \bigcup_{2} e^{9} \cup e^{11} \longrightarrow S^{11}$ is the projection. From (2.6), we have the following exact sequence

$$
0 \longrightarrow \pi_{14}\left(S^{8} \bigcup_{2} e^{9}: 2\right) \stackrel{i^{*}}{\longrightarrow} \pi_{14}\left(S^{8} \bigcup_{2} e^{9} \cup e^{11}: 2\right) \stackrel{j_{*}}{\longrightarrow} \pi_{14}\left(S^{11}: 2\right) \longrightarrow 0 \text {. }
$$

Then there exists a coextension (in the sense of [11]) $\tilde{\nu}_{10}$ of $\nu_{10}$ and $j_{*} \tilde{\nu}_{10}=\nu_{11}$. Assume that $8 \tilde{\nu}_{10}=0$, then $-i_{*} \nu_{8}^{2}=i_{*} \nu_{8}^{2}=8 \tilde{\nu}_{10}$. Let $f: S^{14} \bigvee S^{11}$ $\longrightarrow S^{8} \cup e^{9} \cup e^{11}$ be a map such that $f \mid S^{14}$ and $f \mid S^{11}$ representative of $8 \iota_{14} \oplus \nu_{11}$, then $f \circ g: S^{14} \longrightarrow S^{8} \bigcup_{2} e^{9} \cup e^{11}$ is homotopic to zero. Consider a mapping cone $C_{f}$ of $f$, then there exists a coextension $G: S^{15} \longrightarrow C_{f}$ of $g$. Let $K$ be a mapping cone of $G$, then we have a complex

$$
K=S^{8} \cup e_{6} \cup e^{11} \cup e^{12} \cup e^{15} \cup e^{16}
$$

and $S q^{4} u_{8}=u_{12}, S q^{4} u_{12}=u_{16}$, where $u_{8}, u_{12}$ and $u_{16}$ are cohomology classes mod 2 which are represented by $S^{8}, e^{12}$ and $e^{16}$ respectively. Thus it is verified that $S q^{4} S q^{4} u_{8} \neq 0$ in $K$. By use of Adem's relation

$$
S q^{4} S q^{4} u_{8}=S q^{6} S q^{2} u_{8}+S q^{2} S q^{6} u_{8} \text {. }
$$

Since there is no cell of dimension 10 or 14 in $K$, the right side of the above equation vanishes in $K$, but this is a contradiction. Thus we have proved that $8 \tilde{\nu}_{10}=0$. Therefore, from the exact sequence (2.7), we obtain

$$
\pi_{14}\left(S^{8} \cup e^{9} \cup e^{11}: 2\right)=\left\{i_{*} \nu_{8}^{2}\right\}+\left\{\tilde{\nu}_{10}\right\} \approx Z_{2}+Z_{8} .
$$

In the complex $M=S^{8} \bigcup_{2} e^{9} \cup e^{11} \cup e^{15}$, let $e^{15}$ be attached to $S^{8} \bigcup_{2} e^{9} \cup e^{11}$ by a map $h: S^{14} \longrightarrow S^{8} \bigcup_{2} e^{9} \cup e^{11}$, then we have the sequence

$$
\pi_{14}\left(S^{14}: 2\right) \stackrel{h_{*}}{\longrightarrow} \pi_{14}\left(S^{8} \cup \underset{2}{\cup} e^{9} \cup e^{11}: 2\right) \longrightarrow \pi_{14}(M: 2) \longrightarrow \pi_{14}\left(S^{15}: 2\right)=0
$$


is exact. By Lemma 5.5 of $[10], \quad \pi_{14}\left(F_{4}\right)=Z_{2}$. Thus $\pi_{14}(M: 2) \approx Z_{2}$ and

$$
h_{*} \ell_{14}=b \tilde{\nu}_{10}+a\left(i_{*} \nu_{8}^{2}\right) \quad \text { where } a=0 \text { or } 1 \text {, }
$$

for an odd integer $b$. Thus

$$
j_{*} h_{* \ell_{14}}=\nu_{11} \bmod 2 \pi_{14}\left(S^{11}\right) \text {. }
$$

By theorem 2.6, we have the following important lemma.

Lemma 2. 7. $S q^{4} y_{11}=y_{15}$ in $\tilde{F}_{4}$.

Considering the natural inclusions $\widetilde{F}_{4} \subset \widetilde{E}_{6} \subset \widetilde{E}_{7}$, we have

Corollary 2.8. $S q^{4} y_{11}=y_{15}$ in $\tilde{E}_{6}$ and $\tilde{E}_{7}$.

\section{$\S 3$. Homotopy group of some cell complexes.}

Let $X$ be an $m$-connected $C W$-complex and let $\alpha$ be an element of $\pi_{n-1}(X)(n>m)$. Consider a $C W$-complex $K_{\alpha}=X \cup e^{n}$.

Lemma 3. 1. Let $i$ be an injection $X \longrightarrow K_{\alpha}$ and let $p: K_{\alpha} \longrightarrow S^{n}$ be a mapping which shrinks $X$ to a point. Then the following sequence is exact for $j \leqslant m+n-1$

$$
\cdots \longrightarrow \pi_{j}\left(S^{n-1}\right) \stackrel{\alpha_{*}}{\longrightarrow} \pi_{j}(X) \stackrel{i_{*}}{\longrightarrow} \pi_{j}\left(K_{\alpha}\right) \longrightarrow \pi_{j-1}\left(S^{n-1}\right) \stackrel{\alpha_{*}}{\longrightarrow} \pi_{j-1}(X) \longrightarrow \cdots
$$

Here $\partial$ is a composition $E^{-1} \circ p_{*}: \pi_{j}\left(K_{\alpha}\right) \longrightarrow \pi_{j-1}\left(S^{n-1}\right), \quad$ and $E: \pi_{j-1}\left(S^{n-1}\right)$ $\longrightarrow \pi_{j}\left(S^{n}\right)$ is the suspension homomorphism. If $\alpha$ is of order a power of 2 , then the above sequence is exact for the 2-primary components.

Proof. See Blakers-Massey [4].

We introduce necessary results on the homotopy group of spheres. According to [11], the results are listed in the following table;

(i) $n>k+1$

\begin{tabular}{|c|c|c|c|c|c|c|c|c|c|}
\hline$k=$ & 0 & 1 & 2 & 3 & 4 & 5 & 6 & 7 & 8 \\
\hline$\pi_{n+k}\left(S^{n}: 2\right)$ & $Z$ & $Z_{2}$ & $Z_{2}$ & $Z_{8}$ & 0 & 0 & $Z_{2}$ & $Z_{16}$ & $Z_{2}+Z_{2}$ \\
\hline Generator & $\iota_{n}$ & $\eta_{n}$ & $\eta_{n}^{2}$ & $\nu_{n}$ & & & $\nu_{n}^{2}$ & $\sigma_{n}$ & $\bar{\nu}_{n}, \varepsilon_{n}$ \\
\hline
\end{tabular}

\begin{tabular}{|c|c|c|c|c|c|}
\hline$k=$ & 9 & 10 & 11 & 12 & 13 \\
\hline$\pi_{n+k}\left(S^{n}: 2\right)$ & $Z_{2}+Z_{2}+Z_{2}$ & $Z_{2}$ & $Z_{8}$ & 0 & 0 \\
\hline Generator & $\nu_{n}^{3}, \eta_{n} \varepsilon_{n+1}, \mu_{n}$ & $\eta_{n} \mu_{n+1}$ & $\zeta_{n}$ & & \\
\hline
\end{tabular}


(ii) $n \leqslant k+1 \quad n=9,10,11,13,14$.

(3. 3)

\begin{tabular}{|c|c|c|c|c|}
\hline$k=$ & 8 & 9 & 10 & 11 \\
\hline$\pi_{k+9}\left(S^{9}: 2\right)$ & $Z_{2}+Z_{2}+Z_{2}$ & $Z_{2}+Z_{2}+Z_{2}+Z_{2}$ & $Z_{8}+Z_{2}$ & $Z_{8}+Z_{2}$ \\
\hline Generator & $\sigma_{9} \eta_{16}, \bar{\nu}_{9}, \varepsilon_{9}$ & $\sigma_{9} \eta_{10}^{2}, \nu_{9}^{3}, \mu_{9}, \eta_{9} \varepsilon_{10}$ & $\sigma_{9} \nu_{16}, \eta_{9} \mu_{10}$ & $\zeta_{9}, \bar{\nu}_{9} \nu_{17}$ \\
\hline$\pi_{k+10}\left(S^{10}: 2\right)$ & & $Z+Z_{2}+Z_{2}+Z_{2}$ & $Z_{4}+Z_{2}$ & $Z_{8}$ \\
\hline Generator & & $\Delta\left(\iota_{21}\right), \nu_{10}^{3}, \mu_{10}, \eta_{10} \varepsilon_{11}$ & $\sigma_{10} \nu_{17}, \eta_{10} \mu_{11}$ & $\zeta_{10}$ \\
\hline$\pi_{k+11}\left(S^{11}: 2\right)$ & & & $Z_{2}+Z_{2}$ & $Z_{8}$ \\
\hline Generator & & & $\sigma_{11} \nu_{18}, \eta_{11} \mu_{12}$ & $\zeta_{11}$ \\
\hline$\pi_{k+13}\left(S^{13}: 2\right)$ & & & & \\
\hline Generator & & & & \\
\hline$\pi_{k+14}\left(S^{14}: 2\right)$ & & & & \\
\hline Generator & & & & \\
\hline
\end{tabular}

\begin{tabular}{|c|c|c|c|}
\hline$k=$ & 12 & 13 & 14 \\
\hline$\pi_{k+9}\left(S^{9}: 2\right)$ & 0 & $Z_{2}$ & $Z_{16}+Z_{4}$ \\
\hline Generator & & $\sigma_{9} \nu_{18}^{2}$ & $\sigma_{9}^{2}, \kappa_{9}$ \\
\hline$\pi_{k+10}\left(S^{10}: 2\right)$ & $Z_{4}$ & $Z_{2}$ & $Z_{16}+Z_{2}$ \\
\hline Generator & $\Delta\left(\nu_{21}\right)$ & $\sigma_{10} \nu_{17}^{2}$ & $\sigma_{10}^{2}, \kappa_{10}$ \\
\hline$\pi_{k+11}\left(S^{11}: 2\right)$ & $Z_{2}$ & $Z_{2}+Z_{2}$ & $Z_{16}+Z_{2}$ \\
\hline Generator & $\theta^{\prime}$ & $\theta^{\prime} \eta_{23}, \sigma_{11} \nu_{18}^{2}$ & $\sigma_{11}^{2}, \kappa_{11}$ \\
\hline$\pi_{k+13}\left(S^{13}: 2\right)$ & $Z_{2}$ & $Z_{2}$ & $Z_{16}+Z_{2}$ \\
\hline Generator & $E \theta$ & $E \theta \eta_{25}$ & $\sigma_{13}^{2}, \kappa_{13}$ \\
\hline$\pi_{k+14}\left(S^{14}: 2\right)$ & & $Z$ & $Z_{8}+Z_{2}$ \\
\hline Generator & & $\Delta\left(\iota_{29}\right)$ & $\sigma_{14}^{2}, \kappa_{14}$ \\
\hline
\end{tabular}

We shall use the following relations;

(3. 4) $\sigma_{n} \circ \mu_{n+7}=\eta_{n} \circ \sigma_{n+1}=\bar{\nu}_{n}+\varepsilon_{n} \quad$ for $n \geqslant 10$

by Lemma 6.4 of [11], 
(3. 5) $\sigma_{n} \circ \eta_{n+7}^{2}=\eta_{n}^{2} \circ \sigma_{n+2}=\nu_{n}^{3}+\eta_{n} \circ \varepsilon_{n+1}$ for $n \geqslant 10$

by Lemma 6.3 of [11],

(3. 6) $\sigma_{n} \circ \nu_{n+7}=0$ for $n \geqslant 12$

$\nu_{n} \circ \sigma_{n+3}=0$ for $n \geqslant 11$,

$2 \sigma_{10} \circ \nu_{17}=\nu_{10} \circ \sigma_{13} \quad$ by (7.20) of [11],

$\varepsilon_{n} \circ \eta_{n+8}^{2}=\eta_{n}^{2} \circ \varepsilon_{n+2}=0$ for $n \geqslant 9$ by (7. 10) and (7.20) of ]11],

(3. 7) $\sigma_{n} \circ \bar{\nu}_{n+7}=0$ for $n \geqslant 11$ by (10.8) of [11],

$\sigma_{n} \circ \varepsilon_{n+7}=0 \quad$ for $n \geqslant 6$ by Lemma 10.7 of [11],

(3. 8) $\nu_{n} \circ \varepsilon_{n+3}=\nu_{n} \circ \nu_{n+3}=0$ for $n \geqslant 7$ by (7. 17) of [11],

$\nu_{n} \circ \eta_{n+3}=\eta_{n} \circ \nu_{n+1}=0 \quad$ for $n \geqslant 6$ by (5. 9) of [11],

(3. 9) $\nu_{n} \circ \mu_{n+3}=0$ for $n \geqslant 7$ by Theorem 7.6 of [11],

(3. 10) $\Delta\left(\iota_{21}\right) \circ \eta_{19}=2 \sigma_{10} \circ \nu_{17}$ by $(7.21)$ of [11].

Consider a generator $\sigma_{n}$ of $\pi_{n+7}\left(S^{n}: 2\right) \approx Z_{16}$ for $n \geqslant 9$ and a cell complex $K_{\sigma_{n}}=S^{n} \bigcup_{\sigma_{n}} e^{n+8}$. Let $i: S^{n} \longrightarrow K_{\sigma_{n}}$ be the injection.

Proposition 3.2. We have the following tables of the homotopy groups $\pi_{j}\left(K_{\sigma_{n}}: 2\right)$ for $n=9,10,11,14$ and 15, and generator of their 2-primary components.

(3. 11)

\begin{tabular}{|c|c|c|c|c|c|c|c|c|c|}
\hline$j$ & $j \leq 8$ & 9 & 10 & 11 & 12 & 13 & 14 & 15 & 16 \\
\hline$\pi_{j}\left(K_{\sigma_{9}}: 2\right)$ & 0 & $Z$ & $Z_{2}$ & $Z_{2}$ & $Z_{8}$ & 0 & 0 & $Z_{2}$ & 0 \\
\hline Generator & & $i_{* \ell_{9}}$ & $i_{*} \eta_{9}$ & $i_{*} \eta_{9}^{2}$ & $i_{* \nu_{9}}$ & & & $i_{*} \nu_{9}^{2}$ & \\
\hline
\end{tabular}

\begin{tabular}{|c|c|c|c|c|c|c|}
\hline$j$ & 17 & 18 & 19 & 20 & 21 & 22 \\
\hline$\pi_{k}\left(K_{\sigma_{9}}: 2\right)$ & $Z+Z_{2}+Z_{2}$ & $Z_{2}+Z_{2}+Z_{2}$ & $Z_{2}$ & $Z_{8}+Z_{2}$ & 0 & 0 \\
\hline Generator & $\widetilde{16} \iota_{16}, i_{*} \varepsilon_{9}, i_{*} \bar{\nu}_{9}$ & $i_{*} \eta_{9} \varepsilon_{10}, i_{*} \nu_{9}^{3}, i_{*} \mu_{9}$ & $i_{*} \eta_{9} \mu_{10}$ & $i_{*} \zeta_{9}, i_{*} \bar{\nu}_{9} \nu_{17}$ & & \\
\hline
\end{tabular}

(3. 12)

\begin{tabular}{|c|c|c|c|c|c|c|c|c|c|}
\hline$j$ & $j \leq 9$ & 10 & 11 & 12 & 13 & 14 & 15 & 16 & 17 \\
\hline$\pi_{j}\left(K_{\sigma_{10}}: 2\right)$ & 0 & $Z$ & $Z_{2}$ & $Z_{2}$ & $Z_{8}$ & 0 & 0 & $Z_{2}$ & 0 \\
\hline Generator & & $i_{* \ell}$ & $i_{*} \eta_{10}$ & $i_{*} \eta_{10}^{2}$ & $i_{*} \nu_{10}$ & & & $i_{*} \nu_{10}^{2}$ & \\
\hline
\end{tabular}




\begin{tabular}{|c|c|c|c|c|c|c|}
\hline$j$ & 18 & 19 & 20 & 21 & 22 & 23 \\
\hline$\pi_{j}\left(K_{\sigma_{10}}: 2\right)$ & $Z+Z_{2}$ & $Z+Z_{2}+Z_{2}$ & $Z_{2}$ & $Z_{16}$ & $Z_{4}$ & 0 \\
\hline Generator & $\widetilde{16 \iota_{17}}, i_{*} \varepsilon_{10}$ & $i_{*} \Delta\left(\iota_{21}\right), i_{*} \eta_{10} \varepsilon_{11}, i_{*} \mu_{10}$ & $i_{*} \eta_{10} \mu_{11}$ & $\widetilde{4 \nu_{17}}$ & $i_{*} \Delta\left(\nu_{21}\right)$ & \\
\hline
\end{tabular}

(3. 13)

\begin{tabular}{|c|c|c|c|c|c|c|c|c|c|}
\hline$j$ & $j \leq 9$ & 11 & 12 & 13 & 14 & 15 & 16 & 17 & 18 \\
\hline$\pi_{j}\left(K_{\sigma_{11}}: 2\right)$ & 0 & $Z$ & $Z_{2}$ & $Z_{2}$ & $Z_{8}$ & 0 & 0 & $Z_{2}$ & 0 \\
\hline Generator & & $i_{*} \ell_{11}$ & $i_{*} \eta_{11}$ & $i_{*} \eta_{11}^{2}$ & $i_{*} \nu_{11}$ & & & $i_{*} \nu_{11}^{2}$ & \\
\hline
\end{tabular}

\begin{tabular}{|c|c|c|c|c|c|c|c|}
\hline$j$ & 19 & 20 & 21 & 22 & 23 & 24 & 25 \\
\hline$\pi_{j}\left(K_{\sigma_{11}}: 2\right)$ & $Z_{2}+Z$ & $Z_{2}+Z_{2}$ & $Z_{2}$ & $Z_{32}$ & $Z_{2}$ & $Z_{2}$ & $Z_{2}$ \\
\hline Generator & $i_{*} \varepsilon_{14}, \widetilde{16 \iota_{18}}$ & $i_{*} \mu_{11}, i_{*} \eta_{11} \varepsilon_{12}$ & $i_{*} \eta_{11} \mu_{12}$ & $\widetilde{2 \nu_{18}}$ & $i_{*} \theta^{\prime}$ & $i_{*} \theta^{\prime} \eta_{23}$ & $i_{*} \kappa_{11}$ \\
\hline
\end{tabular}

(3. 14)

\begin{tabular}{|c|c|c|c|c|c|c|c|c|c|}
\hline$j$ & $j \leq 13$ & 14 & 15 & 16 & 17 & 18 & 19 & 20 & 21 \\
\hline$\pi_{j}\left(K_{\sigma_{14}}: 2\right)$ & 0 & $Z$ & $Z_{2}$ & $Z_{2}$ & $Z_{8}$ & 0 & 0 & $Z_{2}$ & 0 \\
\hline Generator & & $i_{* \ell_{14}}$ & $i_{*} \eta_{14}$ & $i_{*} \eta_{14}^{2}$ & $i_{* \nu_{14}}$ & & & $i_{*} \nu_{14}^{2}$ & \\
\hline
\end{tabular}

\begin{tabular}{|c|c|c|c|c|c|c|}
\hline$j$ & 22 & 23 & 24 & 25 & 26 & 27 \\
\hline$\pi_{j}\left(K_{\sigma_{14}}: 2\right)$ & $Z+Z_{2}$ & $Z_{2}+Z_{2}$ & $Z_{2}$ & $Z_{64}$ & 0 & $Z$ \\
\hline Generator & $\widetilde{16} \iota_{21}, i_{*} \varepsilon_{14}$ & $i_{*} \mu_{14}, i_{*} \eta_{14} \varepsilon_{15}$ & $i_{*} \eta_{14} \mu_{15}$ & $\widetilde{\nu_{21}}$ & & $i_{*} \Delta\left(\iota_{29}\right)$ \\
\hline
\end{tabular}

(3. 15)

\begin{tabular}{|c|c|c|c|c|c|c|c|c|c|}
\hline$j$ & & 15 & 16 & 17 & 18 & 19 & 20 & 21 & 22 \\
\hline$\pi_{j}\left(K_{\sigma_{15}}: 2\right)$ & 0 & $Z$ & $Z_{2}$ & $Z_{2}$ & $Z_{8}$ & 0 & 0 & $Z_{2}$ & 0 \\
\hline Generator & & $i_{* \ell} \ell_{15}$ & $i_{*} \eta_{15}$ & $i_{*} \eta_{15}^{2}$ & $i_{*} \nu_{15}$ & & & $i_{*} \nu_{15}^{2}$ & \\
\hline
\end{tabular}

\begin{tabular}{|c|c|c|c|c|c|c|}
\hline$j$ & 23 & 24 & 25 & 26 & 27 & 28 \\
\hline$\pi_{j}\left(K_{\sigma_{15}}: 2\right)$ & $Z+Z_{2}$ & $Z_{2}+Z_{2}$ & $Z_{2}$ & $Z_{64}$ & 0 & 0 \\
\hline Generator & $\widetilde{16 \iota_{22}}, i_{*} \varepsilon_{15}$ & $i_{*} \mu_{15}, i_{*} \eta_{15} \varepsilon_{16}$ & $i_{*} \eta_{15} \mu_{16}$ & $\widetilde{\nu_{22}}$ & & \\
\hline
\end{tabular}


Here we denote by $\tilde{\beta}$ an element of $\pi_{i}\left(K_{\sigma_{n}}: 2\right)$ such that $\partial \tilde{\beta}=\beta \in \pi_{i-1}\left(S^{n+7}: 2\right)$ i.e. we may consider that $\tilde{\beta}$ is a coextension of $\beta$.

Proof. Consider the exact sequence

$$
\begin{gathered}
\cdots \longrightarrow \pi_{j}\left(S^{n+7}: 2\right) \stackrel{\sigma_{n_{*}}}{\longrightarrow} \pi_{j}\left(S^{n}: 2\right) \stackrel{i_{*}}{\longrightarrow} \pi_{j}\left(K_{\sigma_{n}}: 2\right) \stackrel{\partial}{\longrightarrow} \pi_{j-1}\left(S^{n+7}: 2\right) \\
\stackrel{\sigma_{n_{*}}}{\longrightarrow} \pi_{j-1}\left(S^{n}: 2\right) \longrightarrow \cdots
\end{gathered}
$$

of (3. 1) for $j \leqslant 2 n+5$. From $\pi_{j}\left(S^{n+7}: 2\right)=0$ for $j \leqslant n+6$ and from the exactness of the above sequence, it follows that

$$
i_{*}: \pi_{j}\left(S^{n}: 2\right) \longrightarrow \pi_{j}\left(K_{\sigma_{n}}: 2\right)
$$

are isomorphisms onto for $j \leqslant n+6$, and $n=9,10,11,14,15$.

It follows from (3. 1) that the sequence

$$
\pi_{n+7}\left(S^{n+7}: 2\right) \stackrel{\sigma_{n_{*}}}{\longrightarrow} \pi_{n+7}\left(S^{n}: 2\right) \stackrel{i_{*}}{\longrightarrow} \pi_{n+7}\left(K_{\sigma_{n}}: 2\right) \stackrel{\partial}{\longrightarrow} \pi_{n+6}\left(S^{n+7}: 2\right)=0
$$

is exact for $n \geqslant 9$. From $\pi_{n+7}\left(S^{n}: 2\right) \approx\left\{\sigma_{n}\right\} \approx Z_{16}$, we have that

$$
\sigma_{n *}: \pi_{n+7}\left(S^{n+7}: 2\right) \longrightarrow \pi_{n+7}\left(S^{n}: 2\right)
$$

is an epimorphism. Thus we obtain $\pi_{n+7}\left(K_{\sigma_{n}}: 2\right)=0$ for $n=9,10,11,14$ and 15.

Consider the exact sequence

$$
\pi_{n+8}\left(S^{n+7}: 2\right) \stackrel{\sigma_{n}}{\longrightarrow} \pi_{n+8}\left(S^{n}: 2\right) \stackrel{i_{*}}{\longrightarrow} \pi_{n+8}\left(K_{\sigma_{n}}: 2\right) \stackrel{\partial}{\longrightarrow} Z=\left\{16 \iota_{n+7}\right\} \longrightarrow 0
$$

of (3. 1) for $n \geqslant 9$. From (3.2), (3.3) and (3.4) we have that

$$
\sigma_{n *}: \pi_{n+8}\left(S^{n+7}: 2\right) \longrightarrow \pi_{n+8}\left(S^{n}: 2\right)
$$

are monomorphisms for $n \geqslant 9$. Thus it follows from the exactness of the above sequence that the table is true for $\pi_{n+8}\left(K_{\sigma_{n}}: 2\right), n=9,10,11,14,15$.

From (3. 17) and the exact sequence (3.1), it follows that the sequence

$$
\pi_{n+9}\left(S^{n+7}: 2\right) \stackrel{\sigma_{n^{*}}}{\longrightarrow} \pi_{n+9}\left(S^{n}: 2\right) \stackrel{i_{*}}{\longrightarrow} \pi_{n+9}\left(K_{\sigma_{n}}: 2\right) \longrightarrow 0
$$

is exact for $n \geqslant 9$. From (3.5), (3.2) and (3. 3), we have that

$$
\sigma_{n^{*}}: \pi_{n+9}\left(S^{n+7}: 2\right) \longrightarrow \pi_{n+9}\left(S^{n}: 2\right)
$$

is monomorphisms for $n \geqslant 9$. Thus we obtain that 


$$
\pi_{n+9}\left(K_{\sigma_{n}}: 2\right) \approx \pi_{n+9}\left(S^{n}: 2\right) /\left\{\sigma_{n} \circ \eta_{n+7}\right\} .
$$

From (3. 18) and the exact sequence (3.1), it follows that the sequence

$$
\pi_{n+10}\left(S^{n+7}: 2\right) \stackrel{a_{n}}{\longrightarrow} \pi_{n+10}\left(S^{n}: 2\right) \stackrel{i_{*}}{\longrightarrow} \pi_{n+10}\left(K \sigma_{n}: 2\right) \longrightarrow 0
$$

is exact for $n \geqslant 9$. From (3. 2), (3.3) and (3.6), it follows that

(3. 19) $\sigma_{9 *}: \pi_{19}\left(S^{16}: 2\right) \longrightarrow \pi_{19}\left(S^{9}: 2\right)$ is a monomorphism,

$\sigma_{n^{*}}: \pi_{n+10}\left(S^{n+7}: 2\right) \longrightarrow \pi_{n+10}\left(S^{n}: 2\right)$ is trivial for $n=14,15$,

the kernel of $\sigma_{10 *}: \pi_{20}\left(S^{17}: 2\right) \longrightarrow \pi_{20}\left(S^{10}: 2\right)$ is

generated by $\left\{4 \nu_{17}\right\}$, and

the kernel of $\sigma_{11^{*}}: \pi_{21}\left(S^{18}: 2\right) \longrightarrow \pi_{21}\left(S^{11}: 2\right)$ is

generated by $\left\{2 \nu_{18}\right\}$.

Thus it follows that the table is true for $\pi_{n+10}\left(K_{\sigma_{n}}: 2\right) n=9,10,11,14$ and 15.

In the stable rangs, we have the exact sequence

$$
0 \longrightarrow \pi_{n+11}\left(S^{n}: 2\right) \stackrel{i_{*}}{\longrightarrow} \pi_{n+11}\left(K_{\sigma_{n}}: 2\right) \stackrel{\partial}{\longrightarrow} \pi_{n+10}\left(S^{n+7}: 2\right) \longrightarrow 0
$$

of (3.1) for $n \geqslant 13$. Moreover we have the following relation in the stable secondary compositions

$$
\begin{aligned}
\zeta & \in<\sigma, 4 \nu, 2 \iota>\bmod 2 G_{11} & & \text { from Lemma 9. } 1 \text { of [11], } \\
& \supset<\sigma, \nu, 8 \iota> & & \text { from Proposition 1. } 2 \text { of [11], }
\end{aligned}
$$

and $\langle\sigma, \nu, 8 \iota\rangle$ is a coset of the subgroup $\sigma \circ G_{4}+8 G_{11}=8 G_{11}$. Thus

$$
\zeta \equiv\left\langle\sigma, \nu, 8 \iota>\bmod 2 G_{11}\right.
$$

where $G_{n}$ is the $n$-th stable homotopy group of the sphere and $\zeta$ is a generator of the 2-components of $G_{11}$.

From Proposition 1.8 of [11], we obtain

$$
\begin{aligned}
i_{*} \xi & =i_{*}<\sigma, \nu, 8 \iota>\quad \bmod 2 i_{*} G_{11} \\
& =-8 \tilde{\nu}
\end{aligned}
$$

where $\tilde{\alpha} \in \pi_{i}\left(K_{\sigma_{n}}: 2\right)$ is a coextension of $\alpha \in \pi_{i-1}\left(S^{n+7}: 2\right)$. Thus, from this and from the exactness of the above sequence it follows that

$$
\pi_{n+11}\left(K_{\sigma_{n}}: 2\right)=\{\tilde{\nu}\}=Z_{64}
$$

for $n \geqslant 13$ 
From (3. 1), (3. 19) and from $\pi_{n+11}\left(S^{n+7}: 2\right)=0$ for $n \geqslant 0$, it follows the next four exact sequences and the commutative diagram

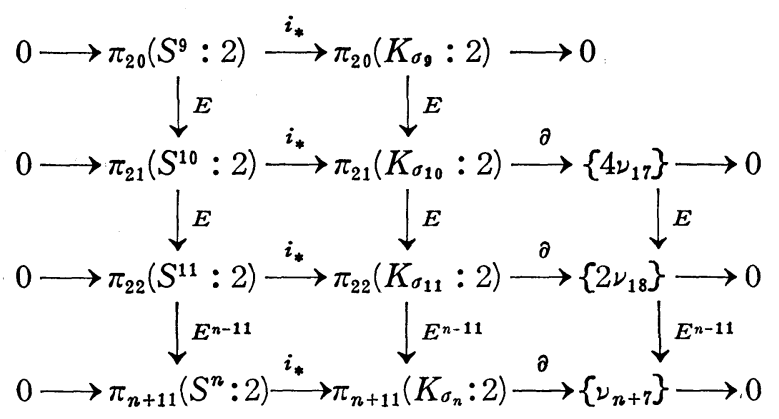

for $\quad n \geqslant 13$, where $E: \pi_{21}\left(S^{10}: 2\right) \longrightarrow \pi_{22}\left(S^{11}: 2\right)$ and $E^{n-11}: \pi_{22}\left(S^{11}: 2\right)$ $\longrightarrow \pi_{n+11}\left(S^{n}: 2\right)$ are isomorphisms. From (3.20) and the above diagram, we obtain that

$$
\begin{aligned}
& \pi_{20}\left(K_{\sigma_{9}}: 2\right)=\left\{i_{*} \zeta_{9}\right\}+\left\{i_{*} \bar{\nu}_{9} \circ \nu_{17}\right\} \approx Z_{8}+Z_{2}, \\
& \pi_{21}\left(K_{\sigma_{11}}: 2\right)=\left\{\tilde{4}_{17}\right\} \approx Z_{16}, \\
& \pi_{22}\left(K_{\sigma_{11}}: 2\right)=\left\{\tilde{2}_{18}\right\} \approx Z_{32}, \\
& \pi_{n+11}\left(K_{\sigma_{n}}: 2\right)=\left\{\tilde{\nu}_{n+7}\right\} \approx Z_{64} \quad \text { for } n \geqslant 13 .
\end{aligned}
$$

It is easily seen the results of $\pi_{n+12}\left(K_{\sigma_{n}}: 2\right)$ and $\pi_{n+13}\left(K_{\sigma_{n}}: 2\right)$ from the exact sequence of (3.1), the table (3.2), (3.3) and the relation (3.6).

Consider the exact sequence

$$
\pi_{25}\left(S^{18}: 2\right) \stackrel{\sigma_{11^{*}}}{\longrightarrow} \pi_{25}\left(S^{11}: 2\right) \stackrel{i^{*}}{\longrightarrow} \pi_{25}\left(K_{\sigma_{11}}: 2\right) \stackrel{\ominus}{\longrightarrow} \pi_{24}\left(S^{18}: 2\right) \stackrel{\sigma_{n^{*}}}{\longrightarrow} \pi_{24}\left(S^{11}: 2\right)
$$

of (3. 1). From (3.2), (3. 3) it follows that

$$
\sigma_{11^{*}}: \pi_{j}\left(S^{18}: 2\right) \longrightarrow \pi_{j}\left(S^{11}: 2\right) \quad \text { for } j=24,25
$$

are monomorphisms. Thus from the exactness of the above sequence we have

$$
\pi_{25}\left(K_{\sigma_{11}}: 2\right) \approx \pi_{25}\left(S^{11}: 2\right) /\left\{\sigma_{11}^{2}\right\}=\left\{\kappa_{11}\right\} \approx Z_{2}
$$

From (3. 1) and (3.2), we have the exact sequence

$$
\pi_{26}\left(S^{18}: 2\right) \stackrel{\sigma_{11^{*}}}{\longrightarrow} \pi_{26}\left(S^{11}: 2\right) \stackrel{i_{*}}{\longrightarrow} \pi_{26}\left(K_{\sigma_{11}}: 2\right) \longrightarrow 0 .
$$

From (3.7) and (3.2), we have that 
3. 22)

$$
i_{*}: \pi_{26}\left(S^{11}: 2\right) \longrightarrow \pi_{26}\left(K_{\sigma_{11}}: 2\right)
$$

an isomorphism onto.

Next consider a generator $\nu_{10}$ of $\pi_{13}\left(S^{10}: 2\right)$ of order 8 and an element $=\Delta\left(\iota_{21}\right)+\gamma$ of $\pi_{19}\left(S^{10}: 2\right)$ of order infinite order, where $\gamma$ is an element $\eta_{20} \circ \varepsilon_{11}+b \nu_{10}^{3}$ of $\pi_{19}\left(S^{10}: 2\right)$ with the order at most $2(a, b=0$ or 1$)$. Let a ell complex $K=S^{11} \cup C\left(S^{13} \vee S^{19}\right)$ be obtained by attaching $C\left(S^{13} \vee S^{19}\right)$ to 10 by $\nu_{10} \vee \beta: S^{13} \bigvee S^{19} \longrightarrow S^{10}$. Then we have the following lemma.

Lemma 3. 3. We have the following table of homotopy group $\pi_{j}(K: 2)$ for $\leqslant 21 ;$

\begin{tabular}{c|c|c|c|c|c|c|c|c|}
\hline$j$ & $j \leqslant 9$ & 10 & 11 & 12 & 13 & 14 & 15 & 16 \\
\hline$\pi_{j}(K: 2)$ & 0 & $Z$ & $Z_{2}$ & $Z_{2}$ & 0 & $Z$ & $Z_{2}$ & $Z_{2}$ \\
\hline Generator & & $i_{*} \ell_{10}$ & $i_{*} \eta_{10}$ & $i_{*} \eta_{10}^{2}$ & & $\widetilde{8 \iota_{13}}$ & $\widetilde{\eta_{13}}$ & $\widetilde{\eta_{13}^{2}}$ \\
\hline$j$ & 17 & 18 & 19 & 20 & 21 \\
\hline$\pi_{j}(K: 2)$ & $Z_{16}+Z_{4}$ & $Z_{2}+Z_{2}$ & $Z_{2}+Z_{2}$ & $Z_{2}+Z_{2}$ & $Z_{128}$ \\
\hline Generator & $i_{*} \sigma_{10}, \widetilde{2 \nu_{13}}$ & $i_{* \nu} \nu_{10}, i_{*} \varepsilon_{10}$ & $i_{*} \eta_{10} \varepsilon_{11}, i_{*} \mu_{10}$ & $i_{*} \sigma_{10} \nu_{17}, i_{*} \eta_{10} \mu_{11}$ & $\widetilde{\sigma_{13} \oplus \eta_{19}}$ \\
\hline
\end{tabular}

Lere $i: S^{10} \longrightarrow K$ is an injection and we denote by $\tilde{\alpha}$ an element of $\pi_{j}(K: 2)$ such hat $\tilde{\alpha}$ is a coextension of $\alpha \in \pi_{j-1}\left(S^{13} \vee S^{19}: 2\right)$.

Proof. By (3. 1), we have an exact sequence

$$
\begin{aligned}
\cdots & \longrightarrow \pi_{j}\left(S^{13} \vee S^{19}: 2\right) \stackrel{\left(\nu_{10} \vee \beta\right)_{*}}{\longrightarrow} \pi_{j}\left(S^{1}{ }_{0}: 2\right) \stackrel{i_{*}}{\longrightarrow} \pi_{j}(K: 2) \\
\stackrel{\partial}{\longrightarrow} \pi_{j-1}\left(S^{13} \vee S^{19}: 2\right) \stackrel{\left(\nu_{10} \vee \beta\right)_{*}}{\longrightarrow} \pi_{j-1}\left(S^{10}: 2\right) \longrightarrow & \longrightarrow
\end{aligned}
$$

or $j \leqslant 21$. We can identify $\pi_{j}\left(S^{13} \vee S^{19}: 2\right)\left(\left(\nu_{10} \vee \beta\right)_{*}\right.$ respectively) with ${ }_{j}\left(S^{13}: 2\right) \oplus \pi_{j}\left(S^{19}: 2\right) \quad\left(\nu_{10^{*}}+\beta_{*}\right.$ respectively) for $j \leqslant 21$ and we shall use the lotation $\alpha=\nu_{10^{*}}+\beta_{*}$.

From the tables (3.2), (3. 3), the relations (3.6), (3. 8) and the exact equence (3. 23), it is easy to see the results of $\pi_{j}(K: 2)$ for $j \neq 17,21$.

Consider the exact sequence

$$
\begin{aligned}
\pi_{17}\left(S^{13}: 2\right) \oplus \pi_{17}\left(S^{19}: 2\right) & \stackrel{\alpha}{\longrightarrow} \pi_{17}\left(S^{10}: 2\right) \stackrel{i_{*}}{\longrightarrow} \pi_{17}(K: 2) \\
& \stackrel{\partial}{\longrightarrow} \pi_{16}\left(S^{13}: 2\right) \oplus \pi_{16}\left(S^{19}: 2\right) \stackrel{\alpha}{\longrightarrow} \pi_{16}\left(S^{10}: 2\right)
\end{aligned}
$$


of (3. 23), where $\pi_{16}\left(S^{13}: 2\right) \oplus \pi_{16}\left(S^{19}: 2\right)=\pi_{16}\left(S^{13}: 2\right)=\left\{\nu_{13}\right\} \approx Z_{8}$ and $\pi_{17}\left(S^{13}: 2\right)$ $\oplus \pi_{17}\left(S^{19}: 2\right)=0$ by (3. 2). We have that the homomorphism $\alpha: \pi_{16}\left(S^{13}: 2\right)$ $\oplus \pi_{16}\left(S^{19}: 2\right) \longrightarrow \pi_{16}\left(S^{10}: 2\right)$ is an epimorphism and its kernal is generated by $\left\{2 \nu_{13}\right\}$. Thus we obtain the following sequence

$$
0 \longrightarrow\left\{\sigma_{10}\right\} \stackrel{i_{*}}{\longrightarrow} \pi_{17}(K: 2) \stackrel{\partial}{\longrightarrow}\left\{2 \nu_{13}\right\} \longrightarrow 0 .
$$

By Adams [1],

$$
\left\{\nu_{10}, 2 \nu_{13}, 4 \iota_{16}\right\} \equiv 0 \quad \bmod 4 \pi_{17}\left(S^{10}: 2\right)
$$

and we have, by Proposition 1.8 of [11], $4 \widetilde{2 \nu_{13}}=-i_{*}\left\{\nu_{10}, 2 \nu_{13}, 4 \iota_{16}\right\}$ $\in 4 i_{*} \pi_{17}\left(S^{10}: 2\right)$. Thus $4\left(\widetilde{2 \nu_{13}}+i_{*} \alpha\right)=0$ for some $\alpha \in \pi_{17}\left(S^{10}: 2\right)$. We may replace $\widetilde{2 \nu_{13}}+i_{*} \alpha$ by $\widetilde{2 \nu_{13}}$. Thus, from (3.24), follows that

$$
\pi_{17}(K: 2)=\left\{i_{*} \sigma_{10}\right\}+\left\{\widetilde{2 \nu_{13}}\right\} \approx Z_{16}+Z_{4} \text {. }
$$

From (3. 23), we have the exact sequence

$$
\begin{aligned}
\pi_{21}\left(S^{13}: 2\right) \oplus \pi_{21}\left(S^{19}: 2\right) \stackrel{\alpha}{\longrightarrow} \pi_{21}\left(S^{10}: 2\right) \stackrel{i_{*}}{\longrightarrow} \pi_{21}(K: 2) \\
\stackrel{\partial}{\longrightarrow} \pi_{20}\left(S^{13}: 2\right) \oplus \pi_{20}\left(S^{19}: 2\right) \stackrel{\alpha}{\longrightarrow} \pi_{20}\left(S^{10}: 2\right) .
\end{aligned}
$$

By (3. 6), (3. 10) and the diagram (3. 2), (3. 3), we have

$$
\alpha\left\{\sigma_{13}\right\}=\nu_{10} \circ \sigma_{13}=2 \sigma_{10} \circ \nu_{17}=\Delta\left(\iota_{21}\right) \circ \eta_{19}=\alpha\left\{\eta_{19}\right\} .
$$

Thus we obtain that

$$
\text { the kernel of } \alpha: \pi_{20}\left(S^{13}: 2\right) \oplus \pi_{20}\left(S^{19}: 2\right) \longrightarrow \pi_{20}\left(S^{10}: 2\right)
$$

is generated by $\left\{\sigma_{13} \oplus \eta_{19}\right\} \approx Z_{16}$.

By (3. 8), (3. 10) and the diagram (3.2),

$$
\begin{aligned}
\alpha\left\{\bar{\nu}_{13}\right\} & =\nu_{10} \circ \bar{\nu}_{13}=0, \\
\alpha\left\{\varepsilon_{13}\right\} & =\nu_{10} \circ \varepsilon_{13}=0, \\
\alpha\left\{\eta_{19}^{2}\right\} & =\beta\left\{\eta_{19}^{2}\right\}=\Delta\left(\iota_{21}\right) \circ \eta_{19}^{2}+a \eta_{10} \circ \varepsilon_{11} \circ \gamma_{19}^{2}+b \nu_{10}^{3} \circ \eta_{19}^{2} \\
& =2 \sigma_{10} \circ \nu_{18} \circ \eta_{19}^{2}+4 a \nu_{10} \circ \varepsilon_{13} \\
& =0 .
\end{aligned}
$$

Thus, from (3. 25), (3. 26) and the from above sequence, it follows that the sequence 


$$
0 \longrightarrow\left\{\zeta_{10}\right\} \stackrel{i_{*}}{\longrightarrow} \pi_{21}(K: 2) \stackrel{\partial}{\longrightarrow}\left\{\sigma_{13} \oplus \eta_{19}\right\} \longrightarrow 0
$$

is exact. By (9.3) of [11],

$$
\zeta_{10} \in\left\{\nu_{10}, 2 \sigma_{13}, 8 \iota_{20}\right\} \quad \bmod 8 \pi_{21}\left(S^{10}: 2\right)
$$

and by Proposition 1.3 of [11]

$$
\begin{aligned}
i_{*} \zeta_{10} & \in i_{*}\left\{\nu_{10}, 2 \sigma_{13}, 8 \iota_{20}\right\} \\
& =-8 \tilde{2 \sigma_{13}} \\
& =-16 \overbrace{\sigma_{13} \oplus \eta_{19}} .
\end{aligned}
$$

Thus we obtain that

$$
\pi_{21}(K: 2)=\left\{\widetilde{\left.\sigma_{13} \oplus \eta_{19}\right\}} \approx Z_{128}\right.
$$

\section{§4. Homotopy groups of exceptional Lie groups $E_{6}, E_{7}$ and $E_{8}$.}

(I) Homotopy GRoups $\pi_{j}\left(E_{8}: 2\right)$ for $j \leqslant 28$.

From Corollary 2.3, Lemma 2.5, there exist a cell complex $K_{\widetilde{E}_{8}}$ $=S^{15} \cup e_{\sigma_{15}}^{23} \cup e^{27} \cup e^{29}$ and a continuous map $f: K_{\widetilde{E}_{8}} \longrightarrow \widetilde{E}_{8}$, from which the following isomorphism $f_{*}$, induced by a map $f$, is obtained;

$$
f_{*}: \pi_{j}\left(S^{15} \bigcup_{\sigma_{15}} e^{23} \cup e^{27} \cup e^{29}: 2\right) \approx \pi_{j}\left(\widetilde{E}_{8}: 2\right) \quad \text { for } j \leqslant 28 .
$$

Let $e^{27}$ be attached to $K_{\sigma_{15}}=S^{15} \cup_{\sigma_{15}} e^{23}$ by a map $g: S^{26} \longrightarrow K_{\sigma_{15}}$ and $e^{29}$ be attached to $S^{15} \cup_{\sigma_{15}} e^{23} \cup e^{27}$ by a map $h: S^{28} \longrightarrow S^{15} \cup_{\sigma_{15}} e^{23} \cup e^{27}$, then, from Corollary 2. 3 and Theorem 2.6, it follows that the next diagrams are commutative
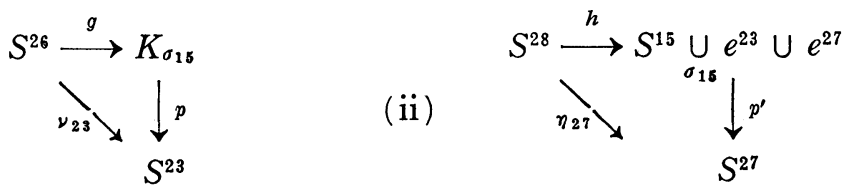

where $p, p^{\prime}$ are the maps which shrink $S^{15}, S^{15} \underset{\sigma_{15}}{\cup} e^{23}$ are respectively to a point. From (4. 1),

$$
\pi_{j}\left(\widetilde{E}_{8}: 2\right) \approx \pi_{j}\left(S^{15} \cup_{\sigma_{16}} e^{23} \cup e^{27}: 2\right) \quad \text { for } j \leqslant 27 .
$$

Consider the exact sequence 


$$
\pi_{26}\left(S^{26}: 2\right) \stackrel{g_{*}}{\longrightarrow} \pi_{26}\left(K_{\sigma_{15}}: 2\right) \stackrel{i_{*}^{\prime}}{\longrightarrow} \pi_{26}\left(S^{15} \bigcup_{\sigma_{15}} e^{23} \cup e^{27}: 2\right) \stackrel{\partial}{\longrightarrow} \pi_{25}\left(S^{26}: 2\right)
$$

of (3. 1), where $i^{\prime}: K_{\sigma_{15}} \longrightarrow S^{15} \cup_{\sigma_{15}} e^{23} \cup e^{27}$ is the inclusion map. From (i) of (4.2) and the table (3. 15), we have that

$$
g_{*}: \pi_{26}\left(S^{26}: 2\right) \longrightarrow \pi_{26}\left(K_{\sigma_{15}}: 2\right)
$$

is an epimorphism. Thus, from the exactness of the above sequence, we obtain

$$
\pi_{26}\left(S^{15} \cup{ }_{\sigma_{15}} e^{23} \cup e^{27}: 2\right)=0 .
$$

It follows from (3. 1), (3. 15) and (4. 3) that the sequence

$$
\begin{gathered}
0=\pi_{27}\left(K_{\sigma_{15}}: 2\right) \stackrel{i_{*}^{\prime}}{\longrightarrow} \pi_{27}\left(S^{15} \bigcup_{\sigma_{15}} e^{23} \cup e^{27}: 2\right) \stackrel{\partial}{\longrightarrow} \pi_{26}\left(S^{26}: 2\right) \\
\stackrel{g_{*}}{\longrightarrow} \pi_{26}\left(K_{\sigma_{15}}: 2\right) \longrightarrow 0
\end{gathered}
$$

is exact. Thus we obtain

$$
\pi_{27}\left(S^{15} \bigcup_{15} e^{23} \cup e^{27}: 2\right)=Z
$$

Next consider the diagram;

$$
\begin{aligned}
& \begin{array}{c}
\pi_{28}\left(S^{15} \cup e_{\sigma_{18}} e^{23}: 2\right)=0 \\
\quad{ }_{i^{i}}^{i_{*}^{\prime}}\left(S^{28}: 2\right) \stackrel{h_{*}}{\longrightarrow} \pi_{28}\left(S^{15} \cup e^{23} \cup e^{27}: 2\right) \stackrel{i_{*}^{\prime \prime}}{\longrightarrow} \pi_{28}\left(S^{15} \cup e^{23} \cup e^{27} \cup e^{29}: 2\right) \stackrel{\partial}{\longrightarrow} \pi_{27}\left(S^{28}: 2\right)=0
\end{array} \\
& \downarrow^{\eta_{27 *}} \stackrel{p_{*}^{\prime}}{\swarrow} \downarrow \downarrow \\
& \pi_{28}\left(S^{27}: 2\right) \underset{E}{\longleftarrow} \pi_{27}\left(S^{26}: 2\right) \\
& \downarrow g_{*} \\
& \pi_{27}\left(S^{15} \bigcup_{\sigma_{15}} e^{23}: 2\right)=0
\end{aligned}
$$

where $i^{\prime \prime}$ is a inclusion map. From (3.1) the row and column sequences are exact, and from (ii) of (4.2) and from the definition of $\partial$, it follows that the diagram is commutative. By (3. 15), $\partial: \pi_{28}\left(S^{15} \cup e^{23} \cup e^{27}: 2\right)$ $\longrightarrow \pi_{27}\left(S^{26}: 2\right)$ is an isomorphism, and $E: \pi_{27}\left(S^{26}: 2\right) \longrightarrow \pi_{28}\left(S^{27}: 2\right)$ is an isomorphism. Thus, from the commutativity of the above diagram, it follows that

$$
h_{*}: \pi_{28}\left(S^{28}: 2\right) \longrightarrow \pi_{28}\left(S^{15} \bigcup_{\sigma_{16}} e^{23} \cup e^{27}: 2\right)
$$


is epimorphic. Thus, from the exactness of the column sequence, we obtain

$$
\pi_{28}\left(S^{15} \bigcup_{\sigma_{15}} e^{23} \cup e^{27} \cup e^{29}: 2\right)=0 .
$$

From (4. 1), (3. 15) and (4. 4) (4. 9), it follows the next table of the homotopy groups of exceptional Lie group $E_{8}$.

Proposition 4. 1 .

\begin{tabular}{|c|c|c|c|c|c|c|c|c|c|}
\hline$j$ & 1,2 & 3 & $4 \leqslant j \leqslant 14$ & 15 & 16 & 17 & 18 & 19 & 20 \\
\hline$\pi_{j}\left(E_{8}: 2\right)$ & 0 & $Z$ & 0 & $Z$ & $Z_{2}$ & $Z_{2}$ & $Z_{8}$ & 0 & 0 \\
\hline$j$ & 21 & 22 & 23 & 24 & 25 & 26 & 27 & 28 \\
\hline$\pi_{j}\left(E_{8}: 2\right)$ & $Z_{2}$ & 0 & $Z+Z_{2}$ & $Z_{2}+Z_{2}$ & $Z_{2}$ & 0 & $Z$ & 0 \\
\hline
\end{tabular}

(II) Hoмотору GRoups $\pi_{j}\left(E_{7}: 2\right)$ for $j \leqslant 25$.

From Lemma 2.5 , there exist a cell complex $K_{\tilde{E}_{7}}=S^{11} \cup e^{15} \cup e^{19} \cup e^{23}$ $\cup e^{26} \cup e^{27}$ and a continuous map $k: K_{\tilde{E}_{7}} \longrightarrow \widetilde{E}_{7}$ such that $k_{*}: \pi_{j}\left(K_{\tilde{E}_{7}}\right)$ $\longrightarrow \pi_{j}\left(\widetilde{E}_{7}\right)$ are $C_{2}$-isomorphism onto for $j \leqslant 28$. By Corollary 2.8 and Lemma 2. 4, $e^{15}$ is attached to $S^{11}$ by a representative of $\nu_{11} \in \pi_{14}\left(S^{11}: 2\right)$.

Consider the diagram

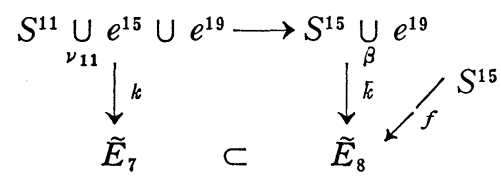

where $p$ is a map which shrinks $S^{11}$ to a point and $\tilde{E}_{7} \subset \tilde{E}_{8}$ is the natural inclusion. Since $\pi_{i}\left(\tilde{E}_{8}\right)=0$ for $i \leqslant 14, k \mid S^{11} \simeq 0$ in $E_{8}$. Thus there exists a map $\quad \bar{k}: S^{15} \cup e^{19} \longrightarrow \widetilde{E}_{8}$ such that the above diagram is homotopy commutative. A generator $x_{15} \in H^{15}\left(\widetilde{E}_{7}: Z_{2}\right)$ corresponds to a generator $x_{15} \in H^{15}\left(\widetilde{E}_{8} ; Z_{2}\right)$ by the natural inclusion $\widetilde{E}_{7} \subset \widetilde{E}_{8}$. Thus, from the commutativity of the above diagram, $x_{15} \in H^{15}\left(\widetilde{E}_{8} ; Z_{2}\right)$ corresponds to a generator of $H^{15}\left(S^{15} \cup e^{19} ; Z_{2}\right)$ by $\bar{k}^{*}$. Let $f: S^{15} \longrightarrow \widetilde{E}_{8}$ be a representative of a generator $\{f\}$ of $\pi_{15}\left(\tilde{E}_{8}\right)=Z$, then $\bar{k} \mid S^{15}$ is homotopic to $x\{f\}$ for some odd integer $x$. Let $e^{19}$ be attached to $S^{15}$ by $\beta: S^{18} \longrightarrow S^{15}$ for a cell complex $S^{15} \cup e^{19}$ of the above diagram.

Since $\bar{k}$ is extended over $e^{19}$, we have

$$
0=\left(\bar{k} \mid S^{15}\right)_{*} \beta=x\left(f_{*} \beta\right) \quad \text { in 2-component. }
$$


By (4. 1$), f_{*}: \pi_{j}\left(S^{15}\right) \longrightarrow \pi_{j}\left(\widetilde{E}_{8}\right)$ are $C_{2}$-isomorphism onto for $j \leqslant 21$. Thus it follows $\beta=0$. From this we have that $S^{11} \cup e^{19}$ is a subcomplex of $K_{\tilde{E}_{7}}$, and $e^{19}$ is attached to $S^{11}$ by $\sigma_{11}$.

LemmA 4. 2. We may regard the inclusion $j: K_{\sigma_{11}}=S^{11} \cup_{\sigma_{11}} e^{19} \subset K_{\tilde{E}_{7}}$ as the fibre map. Let $F$ be the fibre, then $H^{*}\left(F ; Z_{2}\right)$ has additive basis $\left\{1, a_{14}, a_{22}, a_{26}\right\}$ for degree $<29$, where $a_{i}$ denote a generator of degree $i$.

Proof. From lemma 2. 5, $H^{*}\left(K_{\tilde{E}_{7}} ; Z_{2}\right)=\Delta\left(x_{11}, x_{15}, x_{19}, x_{23}, x_{27}\right)$ for degree $<30$ and $S q^{4} x_{11}=x_{15}, S q^{8} x_{15}=x_{23}, S q^{4} x_{23}=x_{27}, S q^{8} x_{11}=x_{19}$. Let $\left\{E_{r}^{* *}\right\}$ be the $\bmod 2$ spectral sequence associated with the above fibering, then we have

$$
E_{2}^{* *}=H^{*}\left(K_{\tilde{E}_{7}} ; Z_{2}\right) \otimes H^{*}\left(F ; Z_{2}\right)
$$

and

$$
E_{\infty}^{* *}=\Delta\left(x_{11}, x_{19}\right) \text { for degree }<30 .
$$

Clearly $K_{\tilde{E}_{7}}$ and $F$ are 10-and 13-connected respectively. We have the following cohomology exact sequence $\cdots \longrightarrow H^{*}\left(K_{\tilde{E}_{7}} ; Z_{2}\right) \stackrel{j^{*}}{\longrightarrow} H^{*}\left(K_{\sigma_{11}} ; Z_{2}\right)$ $\longrightarrow H^{*}\left(F ; Z_{2}\right) \stackrel{\tau}{\longrightarrow} H^{*}\left(K_{\tilde{E}_{7}} ; Z_{2}\right) \longrightarrow \cdots$ for degree $\leqslant 24$. It follows that $H^{*}\left(F ; Z_{2}\right)=\left\{1, a_{14}, a_{22}\right\}$ for degree $<24$ where $\tau\left(a_{14}\right)=x_{15}$ and $\tau\left(a_{22}\right)=x_{23}$, i.e, $\quad d_{15}\left(1 \otimes a_{14}\right)=x_{15} \otimes 1$ and $d_{23}\left(1 \otimes a_{22}\right)=x_{23} \otimes 1$. For $24 \leqslant q \leqslant 29$, any non-zero element of $E_{2}^{0, q}$ must be cancelled by $d_{r}$ with some element of $E_{r}^{r, q-r+1}$. By the dimensional reason, the only posibilities of such $q$ are $q=24,25,26$ corresponding to $x_{11} \otimes a_{14}, x_{11} x_{15} \otimes 1$ and $x_{27} \otimes 1$ respectively. Thus $H^{q}\left(F ; Z_{2}\right)=0$ for $q=27,28,29$. Since $d_{15}\left(x_{11} \otimes a_{14}\right)=x_{11} x_{15} \otimes 1 \neq 0$, $x_{11} \otimes a_{14}$ is not a $d_{15}$-image, hence $H^{24}\left(F ; Z_{2}\right)=0$. We have also $H^{25}\left(F ; Z_{2}\right)$ $=0$ since $x_{11} x_{15} \otimes 1=0$ in $E_{26}^{26,0}$. By the dimensional reason, we see that $x_{27} \otimes 1 \neq 0$ in $E_{27}^{27,0}$, hence there exists an element $a_{26}$ such that $d_{28}\left(1 \otimes a_{26}\right)$ $=x_{27} \otimes 1$ and $a_{26}$ generates $H^{26}\left(F ; Z_{2}\right) \approx Z_{2}$.

From the proof of this lemma, we have that $a_{14}, a_{22}, a_{26}$ are transgressive elements. Since $S q^{8} x_{15}=x_{23}, S q^{4} x_{23}=x_{27}$, it follows, from the commutativity of the Steenrod operation and the transgression, that

$$
S q^{8} a_{14}=a_{22}, \quad S q^{4} a_{22}=a_{26} .
$$

By Lemma 2.5 and Theorem 2.6, there exists a cell complex $K_{F}$ $=S^{14} \cup e^{22} \cup e^{26}$ and a rontinuous map from $K_{F}$ to $F$ which induces 
isomorphisms from $\pi_{j}\left(K_{F}: 2\right)$ onto $\pi_{j}(F: 2)$ for $j \leqslant 26$. Let $f: K_{F} \longrightarrow K_{\sigma_{11}}$ $=S^{11} \cup e^{19}$ be the mapping from a fibre to the total space identifying $F$ with $K_{F}$ for dimension $\leqslant 26$. Then $f \mid S^{14}$ is a representative of $\nu_{11}$.

Consider the exact sequence

$$
\begin{aligned}
& \cdots \longrightarrow \pi_{j}\left(K_{F}: 2\right) \stackrel{f_{*}}{\longrightarrow} \pi_{j}\left(K_{\sigma_{11}}: 2\right) \stackrel{j_{*}}{\longrightarrow} \pi_{j}\left(K_{\tilde{E}_{7}}: 2\right) \\
& \stackrel{\partial}{\longrightarrow} \pi_{j-1}\left(K_{F}: 2\right) \stackrel{f_{*}}{\longrightarrow} \pi_{j-1}\left(K \sigma_{11}: 2\right) \longrightarrow \cdots
\end{aligned}
$$

associated with the above fibering for $j \leqslant 26$ and the following homotopy commutative diagram

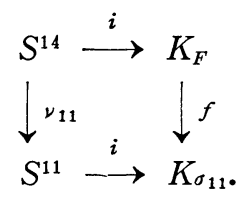

From (3. 1), (3. 14) and from the fact that $e^{26}$ is attached to $K_{\sigma_{14}}$ by a coextension of $\nu_{22}$, we have the next table;

(4. 10)

\begin{tabular}{|c|c|c|c|c|c|c|c|c|}
\hline$j$ & $j \leqslant 13$ & 14 & 15 & 16 & 17 & 18 & 19 & 20 \\
\hline$\pi_{j}\left(K_{F}: 2\right)$ & 0 & $Z$ & $Z_{2}$ & $Z_{2}$ & $Z_{8}$ & 0 & 0 & $Z_{2}$ \\
\hline Generator & & $i_{* \iota_{14}}$ & $i_{*} \eta_{14}$ & $i_{*} \eta_{14}^{2}$ & $i_{*} \nu_{14}$ & & & $i_{*} \nu_{14}^{2}$ \\
\hline
\end{tabular}

\begin{tabular}{|c|c|c|c|c|c|c|}
\hline$j$ & 21 & 22 & 23 & 24 & 25 & 26 \\
\hline$\pi_{j}\left(K_{F}: 2\right)$ & 0 & $Z+Z_{2}$ & $Z_{2}+Z_{2}$ & $Z_{2}$ & 0 & $Z$ \\
\hline Generator & & $\widetilde{16 \iota_{21}}, i_{*} \varepsilon_{14}$ & $i_{*} \mu_{14}, i_{*} \eta_{14} \varepsilon_{15}$ & $i_{*} \eta_{14} \mu_{15}$ & & $\widetilde{64 \iota_{25}}$ \\
\hline
\end{tabular}

Lemma 4. 3. For the homomorphism $f_{*}: \pi_{j}\left(K_{F}: 2\right) \longrightarrow \pi_{j}\left(K_{\sigma_{11}}: 2\right)$, we have the following table;

(4. 11)

\begin{tabular}{|c|c|c|c|c|c|c|c|c|c|c|}
\hline$\alpha=$ & $i_{*} \ell_{14}$ & $i_{*} \eta_{14}$ & $i_{*} \eta_{14}^{2}$ & $i_{*} \nu_{14}$ & $i_{*} \nu_{14}^{2}$ & $i_{*} \widetilde{16 \iota_{21}}$ & $i_{*} \varepsilon_{14}$ & $i_{*} \mu_{14}$ & $i_{*} \eta_{14} \varepsilon_{15}$ & $i_{*} \eta_{14} \mu_{15}$ \\
\hline$f_{*} \alpha=$ & $i_{*} \nu_{14}$ & 0 & 0 & $i_{*} \nu_{14}^{2}$ & $i_{*} \eta_{11} \varepsilon_{12}$ & $4 \widetilde{2 \nu_{18}}$ & 0 & 0 & 0 & 0 \\
\hline
\end{tabular}

Proof. From (4. 9), (3. 8), (3. 9), it follows that the table is true excepting for $\alpha=i_{*} \widetilde{16 \iota_{21}}, i_{*} \nu_{14}^{2}$. 
The relation $i_{*} \eta_{11} \circ \varepsilon_{12}=i_{*} \nu_{11}^{3}$ in $\pi_{20}\left(K \sigma_{11}: 2\right)$ imply the formula

$$
f_{*}\left(i_{*} \nu_{14}^{2}\right)=i_{*} \eta_{11} \circ \varepsilon_{12}
$$

Consider the following commutative diagram

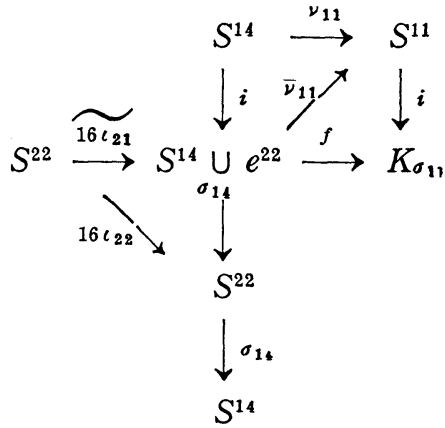

where $\widetilde{16} \iota_{21}$ is a coextension of $16 \iota_{21}$ and $\bar{\nu}_{11}$ is an extension of $\nu_{11}$. We have

$$
\begin{array}{rlrl}
f_{*} \widetilde{\sigma} \iota_{21} & =i_{*} \bar{\nu}_{11} \circ \widetilde{16 \iota_{21}} \\
& =-i_{*}\left\{\nu_{11}, \sigma_{14}, 16 \iota_{21}\right\} & \text { by Proposition } 1.8 \text { of }[11] \\
& =-i_{*} \zeta_{11} & \text { by }(9.3) \text { of }[11] \\
& =-4 \widetilde{\nu}_{21} . &
\end{array}
$$

Proposition 4. 4. The homotopy groups $\pi_{j}\left(E_{7}: 2\right)$ for $j \leqslant 25$ are listed in the following table;

\begin{tabular}{|c|c|c|c|c|c|c|c|c|}
\hline$j$ & 1,2 & 3 & $4 \leqslant j \leqslant 10$ & 11 & 12 & 13 & 14 & 15 \\
\hline$\pi_{j}\left(E_{7}: 2\right)$ & 0 & $Z$ & 0 & $Z$ & $Z_{2}$ & $Z_{2}$ & 0 & $Z$ \\
\hline
\end{tabular}

\begin{tabular}{|c|c|c|c|c|c|c|c|c|}
\hline$j$ & 16 & 17 & 18 & 19 & 20 & 21 & 22 & 23 \\
\hline$\pi_{j}\left(E_{7}: 2\right)$ & $Z_{2}$ & $Z_{2}$ & $Z_{4}$ & $Z+Z_{2}$ & $Z_{2}$ & $Z_{2}$ & $Z_{4}$ & $Z+Z_{2}+Z_{2}$ \\
\hline
\end{tabular}

\begin{tabular}{|c|c|c|}
\hline$j$ & 24 & 25 \\
\hline$\pi_{j}\left(E_{7}: 2\right)$ & $Z_{2}+Z_{2}+Z_{2}$ & $Z_{2}+Z_{2}$ \\
\hline
\end{tabular}

Proof. The results of $\pi_{j}\left(E_{7}: 2\right)$ for $j \leqslant 22$ follow immediately from the tables (4. 10), (3. 13), (4. 11) and from the exactness of the sequence of (4. 8). 


$$
\left\{\nu_{11}, \varepsilon_{14}, 2 \iota_{22}\right\} \supset E^{4}\left\{\nu_{7}, \varepsilon_{10}, 2 \iota_{18}\right\} \subset E^{4} \pi_{19}\left(S^{7}\right)=0 .
$$

Thus we have

$$
\left\{\nu_{11}, \varepsilon_{14}, 2 \iota_{22}\right\} \equiv 0 \quad \bmod 2 \pi_{23}\left(S^{11}\right) .
$$

Similarly we have

$$
\left\{\nu_{11}, \mu_{14}, 2 \iota_{23}\right\} \equiv 0 \quad \bmod 2 \pi_{24}\left(S^{11}\right) .
$$

$$
\left\{\nu_{11}, \eta_{14} \circ \varepsilon_{15}, 2 \iota_{23}\right\} \supset\left\{\nu_{11} \circ \eta_{14}, \varepsilon_{15}, 2 \iota_{23}\right\}=\left\{0, \varepsilon_{15}, 2 \iota_{23}\right\} \equiv 0 \quad \text { by Proposition }
$$

1. 2 of [11]. Thus we have

$$
\left\{\nu_{11}, \eta_{14} \circ \varepsilon_{15}, 2 \iota_{23}\right\} \equiv 0 \quad \bmod 2 \pi_{24}\left(S^{11}: 2\right) .
$$

Similarly,

$$
\left\{\nu_{11}, \eta_{14} \circ \mu_{15}, 2 \iota_{24}\right\} \equiv 0 \quad \bmod 2 \pi_{25}\left(S^{11}: 2\right) .
$$

Consider the commutative diagram

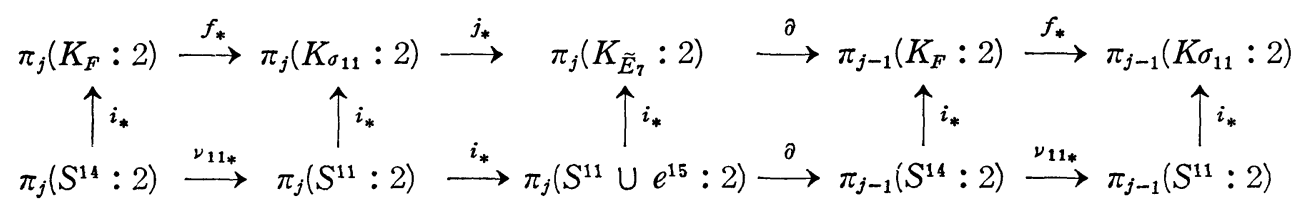

where $i, j$ are inclusions.

From Proposition 1.8 of [11] and the above secondary composition, coextension $\tilde{\varepsilon}_{14}, \tilde{\mu}_{14}, \widetilde{\eta_{14} \circ \varepsilon_{15}}$ and $\widetilde{\eta_{14} \circ \mu_{15}}$ of $\varepsilon_{14}, \mu_{14}, \eta_{14} \circ \varepsilon_{15}$ and $\eta_{14} \circ \mu_{15}$ respectively are elements of order 2 . Thus from the commutativity and the exactness of the above diagram. (4.16), the results of $\pi_{j}\left(K_{E_{7}}: 2\right)$ for $j=23,24,25$, are obtained.

(III) Hoмотору GRoups $\pi_{j}\left(E_{6}: 2\right)$ for $j \leqslant 22$.

By Corollary 2.3,

$$
H^{*}\left(\widetilde{E}_{6} ; Z_{2}\right)=Z_{2}\left[y_{32}\right] \otimes \Delta\left(y_{9}, y_{11}, y_{15}, y_{17}, y_{23}, y_{33}\right)
$$

and

$$
S q^{2} y_{9}=y_{11}, S q^{8} y_{9}=y_{17}, S q^{4} y_{11}=y_{15}, S q^{8} y_{15}=y_{23} .
$$

From Lemma 2. 5, there exists a cell complex $K_{\tilde{E}_{6}}$ and a continuous 
map $l: K_{\tilde{E}_{6}} \longrightarrow \widetilde{E}_{6}$ such that $l_{*}: \pi_{j}\left(K_{\tilde{E}_{6}}\right) \longrightarrow \pi_{j}\left(\widetilde{E}_{6}\right)$ are $C_{2}$-isomorphism onto for $j \leqslant 24$, i.e, $K_{\tilde{E}_{6}}=S^{9} \cup e^{11} \cup e^{15} \cup e^{17} \cup e^{20} \cup e^{23} \cup e^{24}$.

By Corollary 2. 8, $e^{11}$ is attached to $S^{9}$ by $\eta_{9}$.

LemmA 4.5. $K_{\sigma_{9}}=S^{9} \cup e^{17}$ is a subcomplex of $K_{\tilde{E}_{6}}$. Exchanging an inclusion map $K \sigma_{9} \longrightarrow K_{\tilde{E}_{6}}$ by a fibre map, we denote by $F$ the fibre of this fibering. Then $H^{*}\left(F ; Z_{2}\right)$ has the additive basis $\left\{1, a_{10}, a_{14}, a_{20}, a_{22}\right\}$ for degree $\leqslant 25$ such that $S q^{4} a_{10}=a_{14}, S q^{8} a_{14}=a_{22}$, where $a_{i}$ denotes agenerator of degree $i$.

Proof. From Lemma 2. 5, $H^{*}\left(K_{\tilde{E}_{6}} ; Z_{2}\right)=\Delta\left(x_{9}, x_{11}, x_{15}, x_{17}, x_{23}\right)$ for degree $<32$ and $S q^{2} x_{9}=x_{11}, S q^{4} x_{11}=x_{15}, S q^{8} x_{15}=x_{23}, S q^{8} x_{9}=x_{17}$.

By use of Adem's relation we have relations

$$
\begin{aligned}
& S q^{6} x_{11}=S q^{6} S q^{2} x_{9}=S q^{4} S q^{4} x_{9}+S q^{7} S q^{1} x_{9} \\
& S q^{2} x_{15}=S q^{2} S q^{4} x_{11}=S q^{5} S q^{1} x_{11}+S q^{6} x_{11} .
\end{aligned}
$$

Since there is no cell of dimension 10 and $13, S q^{6} x_{11}=0$ in $K \tilde{E}_{6}$. Since there is no cell of dimension 12 and $S q^{6} x_{11}=0, S q^{2} x_{15}=0$ in $K_{\tilde{E}_{6}}$. Then $e^{17}$ is inessential to $e^{15}$, that is, up to homotopy type $S^{9} \cup e^{11} \cup e^{17}$ is a subcomplex. Since $\pi_{16}\left(S^{9} \cup e^{11}, S^{9}\right) \approx \pi_{16}\left(S^{11}\right)=0$, we have that $S^{9} \cup e^{17}$ is a subcomplex. Then, by Theorem 2.6, we may consider that $S^{9} \cup e^{17}=K_{\sigma}$ is a subcomplex of $K_{\tilde{E}_{6}}$.

Let $\left\{E_{r}^{* *}\right\}$ be the mod 2 spectral sequence associated with a fibering $\left\{K_{\sigma_{9}}, i, K_{\tilde{E}_{6}}\right\}$ with the fibre $F$, then

$$
E_{2}^{* *}=H^{*}\left(K_{\tilde{E}_{6}} ; Z_{2}\right) \otimes H^{*}\left(F ; Z_{2}\right)
$$

and

$$
E_{\infty}^{* *}=\wedge\left(x_{9}, x_{17}\right) \quad \text { for degree } \leqslant 25 .
$$

By concerning the cohomology exact sequence associated with this fibering, we have $H^{*}\left(F ; Z_{2}\right)=\left\{1, a_{10}, a_{14}\right\}$ for degree $<18$ with generator $a_{10}, a_{14}$ such that $d_{11}\left(1 \otimes a_{10}\right)=x_{11} \otimes 1$ and $d_{15}\left(1 \otimes a_{14}\right)=x_{15} \otimes 1$. For the total degree $<27, \quad E_{2}^{* *} \quad$ is the sum of $\quad E_{2}^{* *}=H^{*}\left(K_{E_{6}} ; Z_{2}\right) \otimes\left\{1, a_{10}, a_{14}\right\} \quad$ and $\sum_{a \geqslant 18} 1 \otimes H^{q}\left(F ; Z_{2}\right)$. From ' $E_{2}^{* *}$ we compute ' $E_{r}^{* *}$ giving $d_{r}$ trivially except $d_{r}\left(b \otimes a_{10}\right)=b x_{11} \otimes 1$ and $d_{r}\left(b \otimes a_{14}\right)=b x_{15} \otimes 1, b \in H^{*}\left(K_{E_{6}} ; Z_{2}\right)$. Then we have for the total degree $<30,{ }^{\prime} E_{\infty}^{* *}=\Delta\left(x_{9}, x_{17}, x_{23}\right) \otimes 1+\left\{x_{11} \otimes a_{10}, x_{15} \otimes a_{14}\right\}$, where we use the fact $x_{11}^{2}=x_{15}^{2}=0$. Compare this with $E_{\infty}^{* *}$, we conclude that $x_{23} \otimes 1, x_{11} \otimes a_{10}$ must be cancelled by some elements $a_{22}, a_{20}$, i.e, 
$d_{23}\left(1 \otimes a_{22}\right)=x_{23} \otimes 1$ and $d_{11}\left(1 \otimes a_{20}\right)=x_{11} \otimes a_{10} . \quad$ Moreover, no other non-zero elements exists in $H^{*}\left(F ; Z_{2}\right)$ for degree $\leqslant 25$. Thus $H_{*}\left(F ; Z_{2}\right)$ $=\left\{1, a_{10} ; a_{14}, a_{20}, a_{22}\right\}$ for degree $\leqslant 25$.

From the above proof, $a_{10}, a_{14}$ and $a_{22}$ are transgressive element. Since $S q^{4} x_{11}=x_{15}$ and $S q^{8} x_{15}=x_{23}$, using the commutativity of Steenrod operation and transgression we have $S q^{4} a_{10}=a_{14}$ and $S q^{8} a_{14}=a_{22}$.

By Lemma 2.5, there exists a cell complex $K_{F}=S^{10} \cup e^{14} \cup e^{20} \cup e^{22}$ and a continuous map which induce $C_{2}$-isomorphisms from $\pi_{j}\left(K_{F}\right)$ to $\pi_{j}(F)$ for $j \leqslant 24$. We identify the fiber to the total space, then we have a commutative diagram

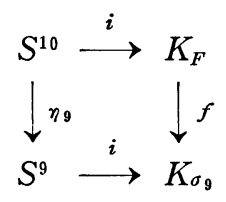

where $i$ is inclusion map, and the exact sequence

$$
\begin{aligned}
\cdots \longrightarrow \pi_{j}\left(K_{F}: 2\right) \longrightarrow \pi_{j}\left(K_{\sigma_{9}}: 2\right) \longrightarrow \pi_{j}\left(K_{\tilde{E}_{6}}: 2\right) & \longrightarrow \pi_{j-1}\left(K_{F}: 2\right) \longrightarrow \pi_{j-1}\left(K_{\sigma_{9}}: 2\right) \longrightarrow \cdots
\end{aligned}
$$

Consider the cell complex $K_{F}=S^{10} \cup e^{14} \cup e^{20} \cup e^{22}$. Since $S q^{4} a_{10}$ $=a_{14}, e^{14}$ is attached to $S^{10}$ by a representative of $\nu_{10}$.

From $\pi_{19}\left(S^{10} \cup e^{14}, S^{10}\right) \approx \pi_{18}\left(S^{13}\right)=0$, we may assume that $K_{F}=S^{10} \mathrm{U}$ $C\left(S^{13} \vee S^{19}\right) \cup e^{22}$.

Let $\alpha: S^{21} \longrightarrow S^{10} \cup C\left(S^{13} \vee S^{19}\right)$ be the attaching map of $e^{22}$ and $e^{20}$ be attached to $S^{10}$ by $\beta: S^{19} \longrightarrow S^{10}$. Consider the exact sequence

$$
\pi_{21}\left(S^{10}: 2\right) \longrightarrow \pi_{21}\left(S^{10} \cup e^{14} \cup e^{20}: 2\right) \stackrel{\partial}{\longrightarrow} \pi_{20}\left(S^{13} \vee S^{19}: 2\right) \stackrel{\left(\nu_{10} \vee \beta\right)_{*}}{\longrightarrow} \pi_{20}\left(S^{10}: 2\right) .
$$

From the definition of $\partial$, we have the commutative diagram

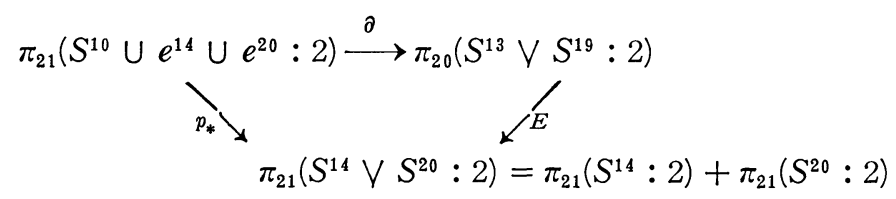

where $p$ is a map which shrinks $S^{10}$ to a point. Since $S q^{8} a_{14}=a_{22}, p_{*} \alpha$ $=\sigma_{14}+x \eta_{20}$ for $x=1$ or 0 . From the exactness of the above sequence, $0=\left(\nu_{10} \vee \beta\right)_{*} \circ \partial \alpha=\nu_{10} \circ \sigma_{13}+x\left(\beta \circ \eta_{19}\right)$. Thus we have $x\left(\beta \circ \eta_{19}\right)=\nu_{10} \circ \sigma_{13} \neq 0$ and $x=1$. 
Put $\beta=a\left(\Delta\left(\iota_{21}\right)\right)+b \eta_{10} \circ \varepsilon_{11}+c \nu_{11}^{3}+d \mu_{10}$ for some integers $a, b, c, d$, then we have

$$
\begin{aligned}
\nu_{10} \circ \sigma_{13} & =\beta \circ \eta_{19} \\
& =a\left(\Delta\left(\iota_{21}\right)\right) \circ \eta_{19}+b \eta_{10}^{2} \circ \varepsilon_{12}+c \nu_{10}^{3} \circ \eta_{19}+d \eta_{10} \circ \mu_{11} \\
& =a \nu_{10} \circ \sigma_{13}+0+0+d \eta_{10} \circ \mu_{11} \quad \text { by }(3.6) \text { and (3. 10). }
\end{aligned}
$$

Thus by (3. 3) $a=1$ and $d=0$. Therefore

$$
\begin{aligned}
& \beta=\Delta\left(\iota_{21}\right)+b \eta_{10} \circ \varepsilon_{11}+c \nu_{10}^{3} \text { where } b, c=0 \text { or } 1 . \\
& \partial \alpha=\sigma_{13}+\eta_{19}
\end{aligned}
$$

From (4. 19), Lemma 3. 3 and from the exact sequence

$\cdots \longrightarrow \pi_{j}\left(S^{21}: 2\right) \stackrel{\alpha_{*}}{\longrightarrow} \pi_{j}\left(S^{10} \cup e^{14} \cup e^{20}: 2\right) \longrightarrow \pi_{j}\left(K_{F}: 2\right) \longrightarrow \pi_{j-1}\left(S^{21}: 2\right) \stackrel{\alpha_{*}}{\longrightarrow} \cdots$ of (3. 1), we have the next table;

(4. 20)

\begin{tabular}{|c|c|c|c|c|c|c|c|c|c|}
\hline$j$ & $j \leqslant 9$ & 10 & 11 & 12 & 13 & 14 & 15 & 16 & 17 \\
\hline$\pi_{j}\left(K_{F}: 2\right)$ & 0 & $Z$ & $Z_{2}$ & $Z_{2}$ & 0 & $Z$ & $Z_{2}$ & $Z_{2}$ & $Z_{16}+Z_{4}$ \\
\hline Generator & & $i_{* \ell_{10}}$ & $i_{*} \eta_{10}$ & $i_{*} \eta_{10}^{2}$ & & $\widetilde{8 \iota_{13}}$ & $\widetilde{\eta_{13}}$ & $\widetilde{\eta_{13}^{2}}$ & $i_{*} \sigma_{10}, \widetilde{2 \nu_{13}}$ \\
\hline
\end{tabular}

\begin{tabular}{|c|c|c|c|c|}
\hline$j$ & 18 & 19 & 20 & 21 \\
\hline$\pi_{j}\left(K_{F}: 2\right)$ & $Z_{2}+Z_{2}$ & $Z_{2}+Z_{2}$ & $Z_{2}+Z_{2}$ & 0 \\
\hline Generator & $i_{*} \overline{\nu_{10}}, i_{*} \varepsilon_{10}$ & $i_{*} \eta_{10} \varepsilon_{11}, i_{*} \mu_{10}$ & $i_{*} \sigma_{10} \nu_{17}, i_{*} \eta_{10} \mu_{11}$ & \\
\hline
\end{tabular}

Lemma 4. 6. For the homomorphism $f_{*}: \pi_{j}\left(K_{F}: 2\right) \longrightarrow \pi_{F}\left(K_{\sigma_{9}}: 2\right)$, we have the following table;

(4. 21)

\begin{tabular}{|c|c|c|c|c|c|c|c|c|}
\hline$\alpha=$ & $i_{*} \ell_{10}$ & $i_{*} \eta_{10}$ & $i_{*} \eta_{10}^{2}$ & $\eta_{13}$ & $i_{*} \sigma_{10}$ & $2 \nu_{13}$ & $i_{*} \nu_{10}$ & $i_{*} \varepsilon_{10}$ \\
\hline$f_{*} \alpha=$ & $i_{*} \eta_{9}$ & $i_{*} \eta_{9}^{2}$ & $4 i_{*} \nu_{9}$ & $i_{* \nu_{9}^{2}}$ & $i_{*} \varepsilon_{9}+i_{* \nu_{9}}$ & $i_{*} \varepsilon_{9}$ & $i_{*} \nu_{9}^{3}$ & $i_{*} \eta_{9} \varepsilon_{10}$ \\
\hline
\end{tabular}

\begin{tabular}{|c|c|c|c|c|}
\hline$\alpha=$ & $i_{*} \eta_{10} \circ \varepsilon_{11}$ & $i_{*} \mu_{10}$ & $i_{*} \sigma_{10} \nu_{17}$ & $i_{*} \eta_{10} \mu_{11}$ \\
\hline$f_{*} \alpha=$ & 0 & $i_{*} \eta_{9} \mu_{10}$ & $i_{* \nu_{9} \nu_{17}}$ & $4 i_{*} \zeta_{9}$ \\
\hline
\end{tabular}

Proof. We shall use the next relations 


$$
\begin{array}{ll}
\eta_{n}^{3}=4 \nu_{n} \quad \text { for } n \geqslant 5 & \text { by }(5.5) \text { of }[11], \\
\eta_{n} \circ \bar{\nu}_{n+1}=\nu_{n}^{3} \quad \text { for } n \geqslant 6 & \text { by Lemma } 6.3 \text { of [11], } \\
\eta_{9} \circ \sigma_{10}=\bar{\nu}_{9}+\varepsilon_{9} & \text { by Lemma } 6.4 \text { of [11], } \\
\eta_{n}^{2} \circ \varepsilon_{n+2}=0 \quad \text { for } n \geqslant 9 & \text { by (7. 10), (7.20) of [11], } \\
4 \zeta_{n}=\eta_{n b}^{2} \circ \mu_{n+2} \quad \text { for } n \geqslant 5 & \text { by Lemma } 6.7 \text { of [11]. }
\end{array}
$$

From (4. 17), (4. 22), it follows that the table is true except for $\alpha=\widetilde{\eta_{13}}$ and $\widetilde{2 \nu_{13}}$.

From the definition of $\widetilde{\eta_{13}}$ and (4.17), we have the commutative diagram

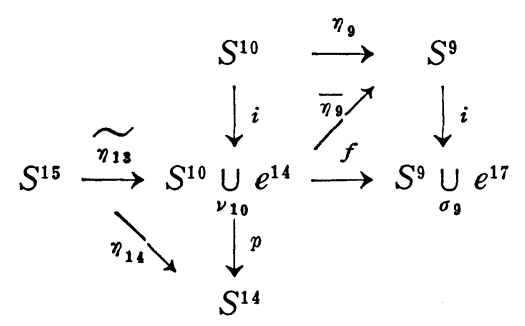

where $p$ is the mapping which shrinks $S^{10}$ to a point and $\overline{\eta_{9}}$ is a extension of $\eta_{9}$. Thus we have

$$
f_{*} \tilde{\eta}_{13}=i_{*} \bar{\eta}_{9} \circ \tilde{\eta}_{13}=i_{*}\left\{\eta_{9}, \nu_{10}, \eta_{13}\right\} \ni i_{*} \nu_{9}^{2} \quad \text { by Lemma } 5.5 \text { of [11]. }
$$

Consider the commutative diagram

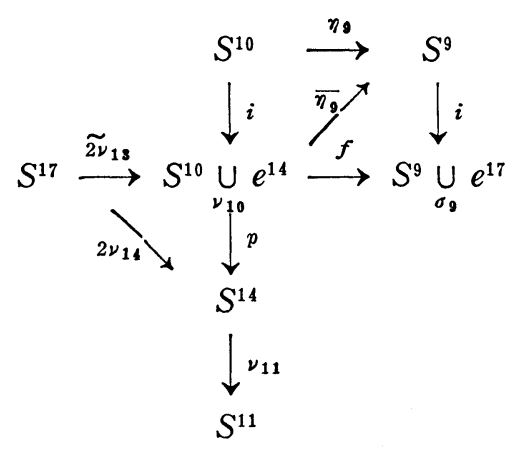

then we have

$$
\begin{aligned}
f_{* 2 \nu_{13}}^{\widetilde{r}}=i_{*} \overline{\eta_{9}} \circ \widetilde{2 \nu_{13}} & \in i_{*}\left\{\eta_{9}, \nu_{10}, 2 \nu_{13}\right\} & & \text { by Proposition } 1.7 \text { of [11], } \\
& \in i_{*} \varepsilon_{9} & & \text { by }(6.1) \text { of [11]. }
\end{aligned}
$$


Proposition 4.7. The homotopy groups $\pi_{j}\left(E_{6}: 2\right)$ for $j \leqslant 22$ are listed in the following table;

\begin{tabular}{|c|c|c|c|c|c|c|c|c|c|}
\hline$j$ & 1,2 & 3 & $4 \leqslant j \leqslant 8$ & 9 & 10 & 11 & 12 & 13 & 14 \\
\hline$\pi_{j}\left(E_{6}: 2\right)$ & 0 & $Z$ & 0 & $Z$ & 0 & $Z$ & $Z_{4}$ & 0 & 0 \\
\hline$j$ & 15 & 16 & 17 & 18 & 19 & 20 & 21 & 22 \\
\hline$\pi_{j}\left(E_{6}: 2\right)$ & $Z$ & 0 & $Z+Z_{2}$ & $Z_{16}+Z_{2}$ & 0 & $Z_{8}$ & 0 & 0 \\
\hline
\end{tabular}

Proof. The results of $\pi_{j}\left(E_{6}: 2\right)$ for $j \neq 18,20$, follow immediately from the table the (3.11), (4. 20), (4. 21) and from the exact sequence (4. 18).

By (3. 9) and Proposition 1. 2 of [11], $\mu \in\langle\eta, 8 \iota, 2 \sigma\rangle \equiv\langle\eta, 2 \sigma, 8 \iota\rangle$ $+\langle 2 \sigma, \eta, 8 \iota\rangle$ and $\langle 2 \sigma, \eta, 8 \iota\rangle \equiv\langle\sigma, 2 \eta, 8 \iota\rangle \equiv 0$. Then, by concerning the suspension homomorphism, we obtain

$$
\left\{\eta_{9}, 2 \sigma_{10}, 8 \iota_{17}\right\} \ni \mu_{9}
$$

By Lemma 9.1 of [11], we have

$$
\left\{\eta_{9}, \eta_{10} \circ \varepsilon_{11}, 2 \iota_{19}\right\} \ni \zeta_{9}
$$

Consider the commutative diagram

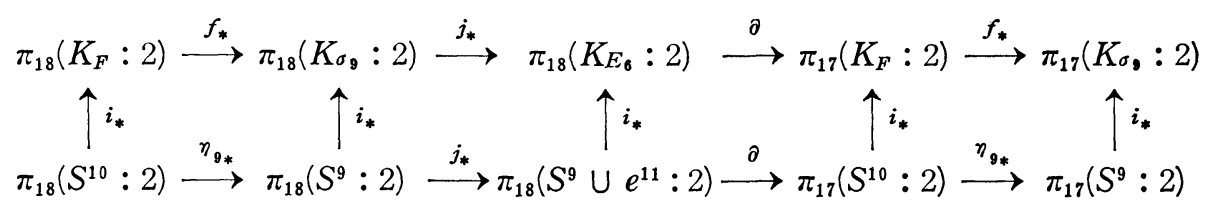

where $j$ is a inclusion map $S^{9} \longrightarrow S^{9} \bigcup_{\eta_{9}} e^{11}$.

By Proposition 1.8 of [11], we have

$$
j_{*} \mu_{9} \in j_{*}\left\{\eta_{9}, 2 \sigma_{10}, 8 \iota_{17}\right\}=-8 \widetilde{2 \sigma_{10}}
$$

From the above commutative diagram and from the tables (3. 11), (4. 20), (4. 21), we obtain

$$
\pi_{18}\left(K_{\widetilde{E_{6}}}: 2\right) \approx Z_{16}+Z_{2} \text {. }
$$

We have the following commutative diagram 


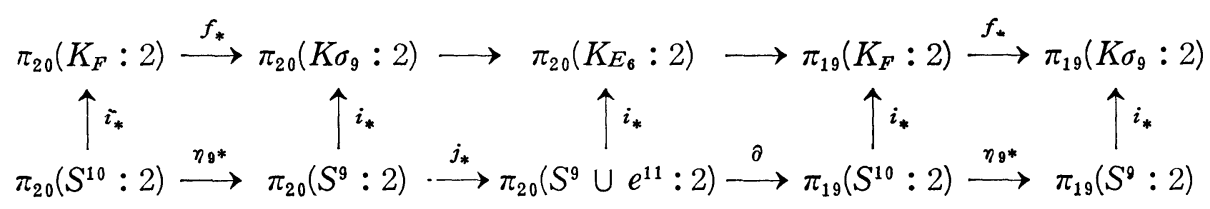

and from Proposition 1.7 of [11]

$$
j_{*} \zeta_{9} \in j_{*}\left\{\eta_{9}, \eta_{10} \circ \varepsilon_{11}, 2 \iota_{19}\right\}=-2 \overbrace{10}^{\circ} \varepsilon_{11} \text {. }
$$

From the exact sequence (4. 18) and from the table (3. 8), (4. 10), (4. 21), we obtain

$$
\pi_{20}\left(K_{\mathbb{E}_{6}}: 2\right) \approx Z_{8}
$$

\section{BIBLIOGRAPHY}

[1] J. F. Adams: On the group $J(X)$ IV, Topology, 5-1 (1966), 21-71.

[2] S. Araki: Cohomology modulo 2 of the compact exceptional groups $E_{6}$ and $E_{7}, \mathrm{~J}$. of Math. Osaka C.V., Vol. 12 (1961), 43-65.

[3] S. Araki and Y. Shikata: Cohomology mod 2 of the compact exceptional group $E_{8}$, Proc. Japan Acad., 37 (1961), 619-622.

[ 4 ] A.L. Blakers and W.S. Massey: The homotopy groups of a triad II, Ann. of Math., 55 (1952), 192-201.

[ 5 ] R. Bott: The stable homotopy of the classical groups, Ann. of Math., 70 (1959), 313-337.

[6] R. Bott and H. Samelson: Application of the theory of Morse to symmetric spaces, Amer. J. Math., 80 (1958), 964-1029.

[ 7 ] H. Cartan and J.P. Serre: Espaces fibrés et groupes d'homotopie I, II, C.R. Acad. Sci. Paris., 234 (1952), 288-290, 393-395.

[ 8 ] J.P. Serre: Groupes d'homotopie et classes de groupes abélian, Ann. of Math., 58 (1953), 258-294.

[ 9 ] J.P. Serre: Cohomologie modulo 2 des complexes d'Eilenberg Mac-Lane, Comm. Math. Helv., 27 (1953), 198-231.

[10] M. Mimura: The homotopy group of Lie groups of low rank, J. Math. Kyoto Univ., 6-2 (1967), 131-176.

[11] H. Toda: Composition methods in homotopy groups of spheres, Ann. of Math. Studies., (1962).

Mathematical Institute

Nagoya University 